\title{
RILIEVO GRAVIMETRICO DELLA SICILIA
}

\author{
E. Medi - C. Morelli
}

1. Generalitì. - Le misure gravimetriche sono state eseguite con i gravimetri Worden n. 50 e n. 52 , previamente studiati $\left({ }^{1}\right)$ e tarati $\left({ }^{2}\right)$. In queste condizioni e purché convenientemente impiegati, essi possono sostituire i pendoli con grandi vantaggi di precisione e di tempo anche nelle operazioni a largo raggrio $\left({ }^{3}\right)$.

Criterio fondamentale è stato perciò sempre quello di operare nelle condizioni richieste per questo tipo di strumenti, cioè principalmente di eseguire tutte le misure almeno in andata e ritorno, e col passo del pellegrino $\mathbf{i}$ collegamenti della rete del $\mathbf{l}^{\circ}$ ordine $(v$. $\$ 2$ ). Inoltre si è cercato di operare fra due stazioni in andata e ritorno possibilmente nello stesso intervallo di tempo e nelle stesse condizioni (di disagio, dato il pessimo fondo di gran parte delle strade di campagna). Data la buona sistemazione già sperimentata $\left({ }^{4}\right)$, le misure sono state tutte effettuate con l'automezzo appositamente attrezzato dell'Osservatorio Geofisico di Trieste.

Le operazioni per la rete del $1^{\circ}$ ordine $(\S 2)$ sono state eseguite dal 28 fehbraio al 15 marzo 1952, quelle per il $2^{\circ}$ ordine e di dettaglio dal 17 marzo al 16 maggio 1952. Complessivamente sono stati percorsi in Sicilia $20.187 \mathrm{~km}$ di maochina.

La scelta dei posti per le misure gravimetriche è vincolata, com'è noto, dalla conoscenza della quota. Le stazioni gravimetriche sono state perciò appoggiate, per quanto possibile, ai caposaldi (verticali: cs. v. IGM; o orizzontali : cs. o. IGM) della livellazione geometrica dell'Istituto Geografico Militare. Poiché però le linee di livellazione in Sicilia non sono molto estese, ovunque era possibile si è fatto riferimento al piano del ferro (p.d.f.) delle ferrovie nelle stazioni (dove ciò̀ l'attendibilità dei dati è maggiore): i controlli eseguiti nei punti in cui esistevano livellazioni di precisione hanno difatti dimostrato che in generale l'errore è inferiore a $0,50 \mathrm{~m}$ (in un solo caso esso ha raggiunto $0,88 \mathrm{~m}$ ). 
Dove neanche la rete ferroviaria era sufficiente per ottenere la densità richiesta (v. $\S 4$ ), si è cercato di riferirsi :

- a punti trigonometrici dell'I.G.M., per i quali la quota del segnale fosse nota da livellazione trigonometrica (l'errore medio è qui molto variabile da zona a zona; in genere, esso non supera $+0,5 \mathrm{~m}$, ma può raggiungere valori anche molto maggiori, specie in montagna);

- a punti quotati dell'I.G.M., dove i trigonometrici mancavano (errore in genere non superiore al $\mathbf{m}$ );

- direttamente al livello del mare $(l . m . m$.$) , per le stazioni lungo$ la costa, dato che le maree sulle coste sicule non sono superiori a $30 \mathrm{~cm}$.

La taratura dei gravimetri è stata controllata all'inizio ed alla fine della campagna sulla base di Catania $\left({ }^{3}\right)$, col seguente risultato:

\begin{tabular}{|c|c|c|c|}
\hline \multirow{2}{*}{ Stazioni } & \multicolumn{2}{|c|}{$\Delta g$ osservato (mgal) } & \multirow{2}{*}{$\Delta$} \\
\hline & 1952 & 1951 & \\
\hline $\begin{array}{l}\text { Catania int. }\left(^{*}\right) \text {. } \\
\text { Catania est. . . } \\
\text { Mascalucia est. . } \\
\text { Mascalucia int. }{ }^{(*)}\end{array}$ & $\begin{array}{l}-0.76+0.01 \\
-100.13+0.02 \\
+\quad 1.29+0.01\end{array}$ & $-99.66 \pm 0.02$ & +0.06 \\
\hline
\end{tabular}

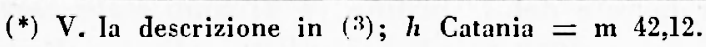

In vista poi della istituzione (mediante misure pendolari) di una comoda base di taratura per gravimetri a grande scala su un forte salto di quota, che estendesse vieppiù verso Sud la serie delle basi italiane $\left({ }^{3}\right)$, la base ora descritta dell'Etna è stata prolungata fino alla Casa Cantoniera, col seguente risultato:

\begin{tabular}{|c|c|}
\hline Stazioni & $\Delta g$ osservato (mgal) \\
\hline Mascalucia est. . . . - & $-313.90+0.03$ \\
Casa Cantoniera est. . - & $-0.02+0.01$ \\
Casa Cantoniera int. (**) & - \\
\hline
\end{tabular}

(**) Sulla soglia della porta, al centro del ballatoio; $h=\mathrm{m} 1882,0$. 
2. Rete di $1^{o}$ ordine. - Disponendo già in Sicilia di due caposaldi della rete fondamentale italiana, dato che il lato Palermo-Catania fa parte del quadrilatero completo compensato dell'Italia merid. $\left({ }^{3}\right)$, si è assunto questo lato come base, e su esso si è appoggiata la triangolazione gravimetrica di $1^{\circ}$ ordine (fig. 1). I collegamenti che la co-

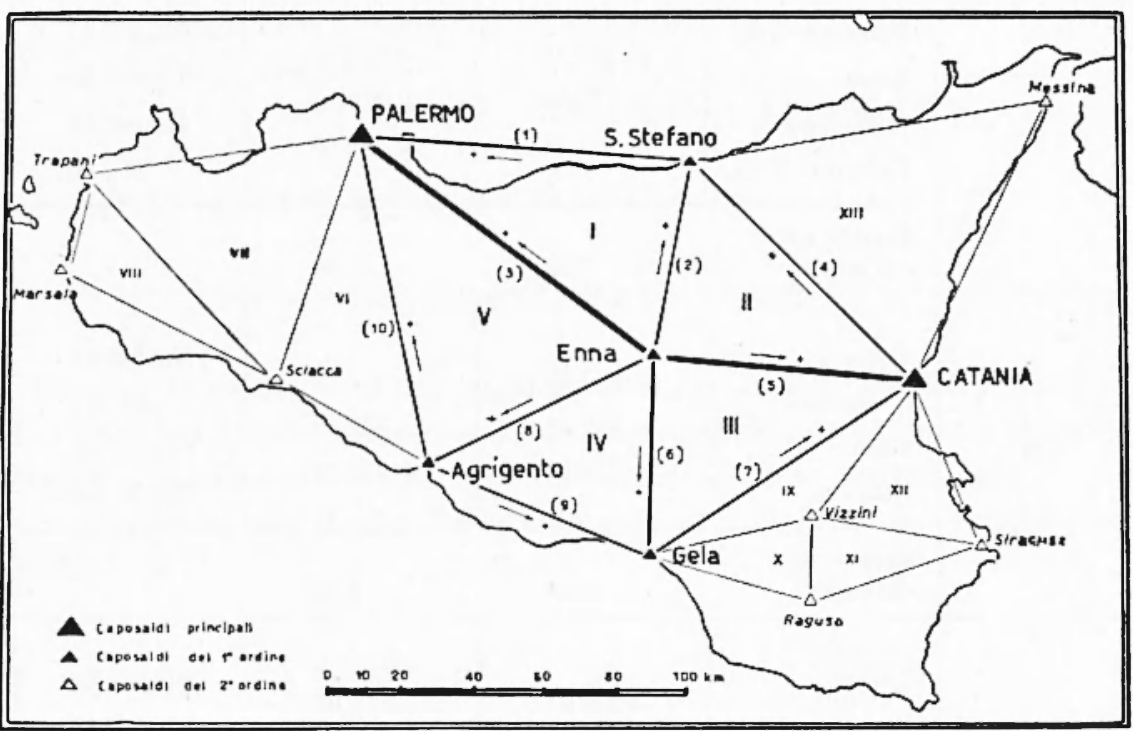

Fig. 1 - Rete gravimetrica del $1^{0}$ e $2^{\text {n }}$ ordine, e schema dei simboli per la compensazione della rete del $1^{\circ}$ ordine

stituiscono sono stati tutti eseguiti almeno due volte, ed i risultati osservati e compensati sono riportati nella Tabella $I$.

La compensazione del pentagono con un punto centrale formato dalla rete del $1^{\circ}$ ordine è stata eseguita col metodo dei minimi quadrati, secondo quanto andiamo ora ad esporre.

Con riferimento alla fig. 1, indichiamo con:

(1),.., (10) i lati dei 5 triangoli che costituiscono il pentagono col punto centrale;

$\Delta_{1}, \ldots, \Delta_{10}$ le medie delle differenze di gravità (in mgal) osservate per ciascun lato, riportate nella Tabella I (la freccia indica il verso della gravità crescente);

$p_{1}, \ldots, p_{10}$ i loro pesi (assunti uguali al numero dei collegamenti di ciascun lato);

$x_{1}, \ldots, x_{10}$ le correzioni da calcolare per ciascuna differenza di gravità osservata. 
Tabella I - Rete di $1^{n}$ ordine

\begin{tabular}{|c|c|c|c|c|}
\hline \multirow{2}{*}{ Triangolo } & \multirow{2}{*}{ Lato } & \multicolumn{2}{|c|}{$\Lambda_{\mathrm{g}}(m g a l)$} & \multirow{2}{*}{$\begin{array}{c}g \\
(\mathrm{~cm} \text { leer }\end{array}$} \\
\hline & & osservato & compensato & \\
\hline \multirow[t]{2}{*}{ I } & $\begin{array}{l}\text { Palermo V.G. } \\
\text { Enna } \\
\text { S. Stefano } \\
\text { Palermo V.G. }\end{array}$ & $\begin{array}{r}-289.18 \\
+279.54 \\
+\quad 9.96\end{array}$ & $\begin{array}{r}-289.55 \\
+279.55 \\
+\quad 10.00\end{array}$ & $\begin{array}{r}980,057.10 \\
979,767.85 \\
980,047.40 \\
, 057.40\end{array}$ \\
\hline & $\begin{array}{l}\text { Errore di } \\
\text { chiusura: }\end{array}$ & $+\quad 0.02$ & 0.00 & \\
\hline \multirow[t]{2}{*}{ II } & $\begin{array}{l}\text { Catania } \\
\text { S. Stefano } \\
\text { Enna } \\
\text { Catania }\end{array}$ & $\begin{array}{l}+\quad 1.45 \\
-279.5 .1 \\
+278.07\end{array}$ & $\begin{array}{l}+\quad 1.52 \\
-279.55 \\
+278.03\end{array}$ & $\begin{array}{r}980,0.45 .88 \\
, 0.17 .40 \\
979,767.85 \\
980,0.15 .88\end{array}$ \\
\hline & $\begin{array}{l}\text { Errore di } \\
\text { chiusura: }\end{array}$ & $-\quad 0.02$ & 0.00 & \\
\hline \multirow[t]{2}{*}{ III } & $\begin{array}{l}\text { Catania } \\
\text { Enna } \\
\text { Gela } \\
\text { Catania }\end{array}$ & $\begin{array}{l}-278.07 \\
+177.61 \\
+100.37\end{array}$ & $\begin{array}{l}-278.03 \\
+177.71 \\
+100.32\end{array}$ & $\begin{array}{r}980,0.45 .88 \\
979,767.85 \\
, 9.45 .56 \\
, 0.45 .88\end{array}$ \\
\hline & $\begin{array}{l}\text { Errore di } \\
\text { chiusura: }\end{array}$ & $-\quad 0.09$ & 0.00 & \\
\hline \multirow[t]{2}{*}{ IV } & $\begin{array}{l}\text { Enna } \\
\text { Agrigento } \\
\text { Gela } \\
\text { Enna }\end{array}$ & $\begin{array}{r}+\quad 79.20 \\
+\quad 98.78 \\
-177.61\end{array}$ & $\begin{array}{r}+\quad 79.08 \\
+\quad 98.63 \\
-177.71\end{array}$ & $\begin{array}{r}979,767.85 \\
, 8+6.93 \\
, 915.56 \\
, 767.85\end{array}$ \\
\hline & $\begin{array}{l}\text { Errore di } \\
\text { chiusura: }\end{array}$ & $+\quad 0.37$ & 0.00 & \\
\hline \multirow[t]{2}{*}{$V$} & $\begin{array}{l}\text { Palermo V.G. } \\
\text { Agrigento } \\
\text { Enna } \\
\text { Palermo V.G. }\end{array}$ & $\begin{array}{r}-210.43 \\
-\quad 79.20 \\
+289.48\end{array}$ & $\begin{array}{l}-210.17 \\
-79.08 \\
+289.55\end{array}$ & $\begin{array}{r}980,057.40 \\
979,846.93 \\
, 767.85 \\
980,057.40\end{array}$ \\
\hline & $\begin{array}{l}\text { Errore di } \\
\text { chiusura: }\end{array}$ & $-\quad 0.15$ & 0.00 & \\
\hline
\end{tabular}


Ne risultano le seguenti equazioni di condizione:

$$
\begin{aligned}
& \left(\Delta_{1}+x_{1}\right)-\left(\Delta_{2}+x_{2}\right)-\left(\Lambda_{3}+x_{33}\right)=0 \\
& -\left(\Delta_{2}+x_{2}\right)+\left(\Delta_{4}-x_{4}\right)+\left(\Delta_{5}+x_{5}\right)=0 \\
& -\left(\Delta_{5}+x_{5}\right)+\left(\Delta_{6}+x_{8}\right)+\left(\Delta_{7}+x_{7}\right)=0 \\
& -\left(\Delta_{i}+x_{6}\right)+\left(\Delta_{8}+x_{8}\right)+\left(\Delta_{9}+x_{9}\right)=0 \\
& \left(\Delta_{3}+x_{3}\right)-\left(\Delta_{8}+x_{8}\right)-\left(\Delta_{10}+x_{10}\right)=0 \\
& -\left(\Delta_{1}+x_{1}\right)-\left(\Delta_{4}+x_{1}\right)-\left(\Delta_{2}+x_{7}\right)-\left(\Lambda_{9}-x_{9}\right)-\left(\Lambda_{10}+x_{10}\right)=0
\end{aligned}
$$

alla quale vogliamo aggiungere la condizione

$$
\left.-\left(\Delta_{3}+x_{3}\right)+(\lrcorner_{5}+x_{5}\right)=-11.52
$$

dato che il valore - 11.52 mgal risulta per il lato Palermo V. G. Catania (est.) dalla compensazione del quadrilatero completo Roma Napoli - Palermo - Catania della rete fondamentale italiana $\left({ }^{3}\right)$.

Poiché, come risulta dalla colonna dei valori osservati della Tabella $I$, ¿̀:

$$
\begin{array}{rlr}
\Delta_{1}+\Delta_{2}-\Delta_{.} & =+0.02 \\
-\Delta_{2}+\Delta_{4}-\Delta_{5} & =-0.02 \\
-\Delta_{5}+\Delta_{2}+\Delta_{1} & =-0.09 \\
-\Delta_{2}+\Delta_{8}+\Delta_{9} & =+0.37 \\
\Delta_{3}-\Delta_{8}-\Delta_{10} & =-0.15 \\
-\Delta_{1}-\Delta_{4}-\Delta_{7}-\Delta_{9}+\Delta_{10} & =-0.13 \\
-\quad-\Delta_{0}+\Delta_{5} & & =-11.41
\end{array}
$$

si ricava:

$$
\begin{aligned}
x_{1}+x_{2}-x_{3}+0.02 & =0 \\
-x_{2}+x_{4}+x_{5}-0.02 & =0 \\
-x_{5}+x_{6}+x_{7}-0.09 & =0 \\
-x_{6}+x_{8}+x_{3}+0.37 & =0 \\
x_{3}-x_{8}-x_{10}-0.15 & =0 \\
-x_{1}-x_{4}-x_{7}-x_{3}+x_{10}-0.13 & =0 \\
-x_{3}+x_{5}+0.11 & =0
\end{aligned}
$$

alle quali hisogna agguiungere la condizione:

$$
\sum_{\mathrm{i}=1}^{10} p_{\mathrm{i}} x_{\mathrm{i}}=\operatorname{minimo}
$$


Disponendo le equazioni di condizione nello specchio seguente:

\begin{tabular}{|c|c|c|c|c|c|c|c|c|c|c|c|c|}
\hline Correl. & Equaz. & $x_{1}$ & $x_{3}$ & $x_{3}$ & $x_{4}$ & $x_{3}$ & $\boldsymbol{x}_{\mathrm{i}}$ & $x_{7}$ & $x_{8}$ & $x_{9}$ & $x_{10}$ & $v$ \\
\hline $\begin{array}{l}\mathbf{K}_{1} \\
\mathbf{K}_{2} \\
\mathbf{K}_{3} \\
\mathbf{K}_{3} \\
\mathbf{K}_{5} \\
\mathbf{K}_{6} \\
\mathbf{K}_{7}\end{array}$ & $\begin{array}{l}\mathbf{A} \\
\mathbf{B} \\
\mathbf{C} \\
\mathbf{D} \\
\mathbf{E} \\
\mathbf{F} \\
\mathbf{G}\end{array}$ & +1 & $\begin{array}{l}+1 \\
-1\end{array}$ & $\begin{array}{l}-1 \\
+1 \\
-1 \\
\end{array}$ & $\begin{array}{l}+1 \\
-1\end{array}$ & $\begin{array}{c}+1 \\
-1 \\
-1\end{array}$ & $\begin{array}{l}+1 \\
-1\end{array}$ & $\begin{array}{l}+1 \\
-1\end{array}$ & $\begin{array}{l}+1 \\
-1\end{array}$ & $\begin{array}{r}+1 \\
-1\end{array}$ & $\frac{-1}{-1}$ & $\begin{array}{l}+0.02 \\
-0.02 \\
-0.09 \\
+0.37 \\
-0.15 \\
-013 \\
+0.11\end{array}$ \\
\hline & $\mathrm{p}$ & 4 & 4 & 2 & 2 & 4 & 2 & 2 & 2 & 2 & 2 & \\
\hline
\end{tabular}

si ricavano facilmente le equazioni correlate, che sono:

$$
\begin{array}{lll}
x_{1}=-1 / 4\left(K_{1}-K_{6}\right) & ; & x_{6}=-1 / 2\left(K_{: 3}-K_{4}\right) \\
x_{2}=-1 / 4\left(K_{1}-K_{.0}\right) & ; & x_{7}=-1 / 2\left(K_{3}-K_{6}\right) \\
x_{3}=-1 / 2\left(-K_{1}+K_{5}-K_{7}\right) & ; & x_{8}=-1 / 2\left(K_{1}-K_{5}\right) \\
x_{4}=-1 / 2\left(K_{2}-K_{6}\right) & ; & x_{9}=-1 / 2\left(K_{1}-K_{63}\right) \\
x_{5}=-1 / 4\left(K_{2}-K_{3}+K_{7}\right) & ; & x_{10}=-1 / 2\left(-K_{5}+K_{6}\right)
\end{array}
$$

Introducendo queste espressioni delle $x$ nelle equazioni di condizione, si ricava il seguente sistema normale di 7 equazioni nelle 7 incognite $K_{\mathrm{i}}(i=1, \ldots, 7)$ :

$$
\begin{aligned}
& 1,0 . K_{1}-0,25 . K_{e}+0 \quad . K_{3}+0 \quad . K_{1}-0,5 . K_{s}-0,25 . K_{i}+0,5 \quad . K_{i}-0,02=0
\end{aligned}
$$

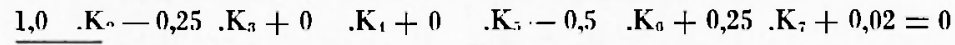

$$
\begin{aligned}
& 1,25 . K_{:}-0,5 . K_{1}+0 \quad . K_{:}-0,5 \quad . K_{i i}-0,25 \quad K_{i}+0,09=0 \\
& \underline{1,5 . K_{i}-0,5 . K_{i}-0,5 \quad . K_{:}+0 \quad . K_{i}-0,37=0} \\
& \underline{1,5 \cdot K_{i}-0,5} \quad K_{i:}-0,5 \quad . K_{i}+0,15=0 \\
& \underline{2,25 \cdot K_{i}+0 \quad . K_{i}+0,13=0} \\
& 0,75 \cdot K_{7}-0,11=0
\end{aligned}
$$

dove i coefficienti della diagonale principale sono la somma dei reciproci dei pesi per ognuno dei lati considerati.

La risoluzione col metodo di Gauss porta al seguente sistema risolvente:

$$
\begin{aligned}
& 1,0 . K_{1}-0,25 \quad . K_{2}+0 \quad . K_{3}+0 \quad . K_{4}-0,5 \quad . K_{-0,25} \quad . K_{t-+10,5} \quad . K_{-0,02}=0 \\
& 0,938 . K_{2}-0,25 \quad . K_{:}+0 \quad . K_{1}-0,125 . K_{:}-0,563 \quad K_{i}+0,375 \quad . K_{:}+0,015=0 \\
& 1,183 . K_{i}-0,500 . K_{4}-0,033 . K_{i-1}-0,650 \quad . K_{i}-0,150 . K_{i}+0,094=0 \\
& 1,289 . \mathrm{K}_{\mathbf{i}}-0,514 . \mathrm{K}_{;}-0,775 . \mathrm{K}_{i-}-0,063 \quad \mathrm{~K}_{i}-0,330=0 \\
& 1,027 . K_{:-1,027} \cdot K_{11}-0,230 \quad . K_{7}+0,013=0 \\
& 0,0001 . K_{i}+-0 \quad . K_{i}-0,0001=0 \\
& 0,2766 \cdot \mathrm{K}-0,107 \cdot 1=0
\end{aligned}
$$


dal quale si ottengono $\mathrm{i}$ seguenti valori per i correlativi $K_{\mathrm{i}}$ :

$$
\begin{array}{lll}
K_{1}=+0,829 & ; & K_{5}=+1,074 \\
K_{\bullet}=+0,866 & ; & K_{1 ;}=+1,000 \\
K_{3}=+1,101 & ; & K_{\bar{\imath}}=+0,388 \\
K_{4}=+1,305 & ; &
\end{array} ;
$$

A comprova dell'esattezza dei calcoli, la sostituzione di questi valori nel sistema normale porta alla seguente somma dei quadrati degli errori di chiusura $V$ delle sue equazioni:

$$
[V V]=0,0000 \text {. }
$$

Sostituendo invece i valori $K_{1}$ sopra ottenuti nelle equazioni correlate, si ricavano $i$ seguenti valori per le correzioni $x_{i}$ da applicarsi alle differenze di gravità osservate:

$$
\begin{aligned}
& x_{1}=+0,04 \mathrm{mgal} ; x_{\mathrm{s}}=-0.04 \mathrm{mgal} ; x_{\mathrm{s}}=-0.12 \mathrm{mgal} ;
\end{aligned}
$$

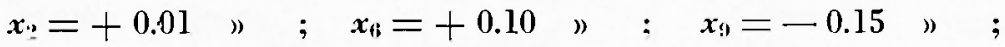

$$
\begin{aligned}
& x_{3}=+0.07 \text { » } ; x_{7}=-0.05 \quad \text { ” } \quad \text { : } x_{10}=+0.04 \text { » . } \\
& x_{\varepsilon}=+0.07 \text { ” ; }
\end{aligned}
$$

Sostituendo questi valori nelle equazioni di condizione, la somma dei quadrati dei residui risulta:

$$
\left[V^{\prime} V^{\prime}\right]=0,0000 \text {. }
$$

Quadrando e sommando i valori ora ottenuti per le correzioni $x_{i}$ si ricava inoltre:

$$
[p x x]=+0.1350,
$$

mentre la stessa sommatoria calcolata per controllo mediante la

$$
[p x x]=-\sum_{i=1}^{z} K_{1} v_{i}
$$

fornisce il valore

$$
[p x x]=+0.1347 \text {, }
$$

in buon accordo col precedente.

Indicando con $q$ il numero delle equazioni di condizione, l'errore medio $m_{o}$ dell'unità di peso, cioè di un collegamento, diventa:

$$
m_{0}= \pm \frac{\sqrt{[p x x]}}{q}=\div 0.14 \text { mgal. }
$$

Questo risultato è soddisfacente, soprattutto se si considerano le disagiate condizioni d'impiego. Esso indica la precisione "interna " dei vertici della rete di $l^{\circ}$ ordine. 
Tabella II - Rete di $2^{\circ}$ ordine

\begin{tabular}{|c|c|c|c|c|}
\hline \multirow{2}{*}{ Triangolo } & \multirow{2}{*}{ Lato } & \multicolumn{2}{|c|}{$\Delta g \quad(m g a l)$} & \multirow{2}{*}{$\begin{array}{c}g \\
\left(\mathrm{~cm} \mid \cdot s e c^{2}\right)\end{array}$} \\
\hline & & osservato & compensato & \\
\hline \multirow[t]{2}{*}{ VI } & $\begin{array}{l}\text { Palermo V.G. } \\
\text { Sciacca } \\
\text { Agrigento } \\
\text { Palermo V.G. }\end{array}$ & $\begin{array}{l}-56.58 \\
-151.02 \\
+210.13\end{array}$ & $\begin{array}{l}-56.58 \\
-153.89 \\
+210.17\end{array}$ & $\begin{array}{r}980,057.40 \\
, 000.82 \\
979,8.16 .93 \\
980,057.40\end{array}$ \\
\hline & $\begin{array}{l}\text { Errore di } \\
\text { chiusura: }\end{array}$ & $-\quad 0.17$ & 0.00 & \\
\hline \multirow[t]{2}{*}{ VII } & $\begin{array}{l}\text { Palermo V.G. } \\
\text { Trapani } \\
\text { Sciacea } \\
\text { Palermo V.G. }\end{array}$ & $\begin{array}{r}+\quad 0.7 .1 \\
-\quad 57.39 \\
+\quad 56.58\end{array}$ & $\begin{array}{r}+\quad 0.78 \\
-\quad 57.36 \\
+\quad 56.58\end{array}$ & $\begin{array}{r}980,057.40 \\
, 058.18 \\
, 000.82 \\
, 057.40\end{array}$ \\
\hline & $\begin{array}{l}\text { Errore di } \\
\text { chiusura: }\end{array}$ & $-\quad 0.07$ & 0.00 & \\
\hline \multirow[t]{2}{*}{ VIII } & $\begin{array}{l}\text { Trapani } \\
\text { Marsala } \\
\text { Sciacea } \\
\text { Trapani }\end{array}$ & $\begin{array}{l}-19.15 \\
-\quad 8.01 \\
+\quad 57.39\end{array}$ & $\begin{array}{r}-\quad 19.10 \\
-\quad 7.96 \\
+\quad 57.36\end{array}$ & $\begin{array}{r}980,058.18 \\
, 008.78 \\
, 0110.82 \\
, 058.18\end{array}$ \\
\hline & $\begin{array}{l}\text { Errore di } \\
\text { chiusura: }\end{array}$ & $-\quad 0.07$ & 0.00 & \\
\hline \multirow[t]{2}{*}{ IX } & $\begin{array}{l}\text { Catania } \\
\text { Gela } \\
\text { Vizzini } \\
\text { Catania }\end{array}$ & $\begin{array}{r}-100.37 \\
-\quad 31.69 \\
+132.06\end{array}$ & $\begin{array}{r}-100.32 \\
-\quad 31.71 \\
+132.03\end{array}$ & $\begin{array}{r}980,0.15 .88 \\
979,915.56 \\
, 913.85 \\
980,015.88\end{array}$ \\
\hline & $\begin{array}{l}\text { Errore di } \\
\text { chiusura: }\end{array}$ & 0.00 & 0.00 & \\
\hline
\end{tabular}


Tabella II - Rele di $2^{\prime \prime}$ ordine

\begin{tabular}{|c|c|c|c|c|}
\hline \multirow{2}{*}{ Triangolo } & \multirow{2}{*}{ Lato } & \multicolumn{2}{|c|}{$\Delta_{\mathrm{g}} \quad(\mathrm{mgal})$} & \multirow{2}{*}{$\begin{array}{c}\text { B } \\
\left.|c m| s e e^{2}\right)\end{array}$} \\
\hline & & osservato & compensato & \\
\hline \multirow[t]{2}{*}{$\mathbf{X}$} & $\begin{array}{l}\text { Gela } \\
\text { Ragusa } \\
\text { Vizzini } \\
\text { Gela }\end{array}$ & $\begin{array}{r}27.02 \\
-\quad 4.69 \\
+\quad 31.69\end{array}$ & $\begin{array}{r}-\quad 27.02 \\
-\quad 4.69 \\
+\quad 31.71\end{array}$ & $\begin{array}{r}979,945.56 \\
, 918.54 \\
, 913.85 \\
, 945.56\end{array}$ \\
\hline & $\begin{array}{l}\text { Errore di } \\
\text { chiusura: }\end{array}$ & $-\quad 0.02$ & 0.00 & \\
\hline \multirow[t]{2}{*}{ XI } & $\begin{array}{l}\text { Vizzini } \\
\text { Ragusa } \\
\text { Siracusa } \\
\text { Vizzini }\end{array}$ & $\begin{array}{r}+\quad 4.69 \\
+134.40 \\
-139.07\end{array}$ & $\begin{array}{r}+\quad 4.69 \\
+134.42 \\
-139.11\end{array}$ & $\begin{array}{r}979,313.85 \\
, 918.54 \\
980,052.96 \\
979,913.85\end{array}$ \\
\hline & $\begin{array}{l}\text { Errore di } \\
\text { chiusura: }\end{array}$ & $+\quad 0.02$ & 0.00 & \\
\hline \multirow[t]{2}{*}{ XII } & $\begin{array}{l}\text { Catania } \\
\text { Vizzini } \\
\text { Siracusa } \\
\text { Cattania }\end{array}$ & $\begin{array}{r}-132.06 \\
+139.07 \\
-\quad 7.12\end{array}$ & $\begin{array}{r}-132.03 \\
+139.11 \\
-\quad 7.02\end{array}$ & $\begin{array}{r}980,0.45 .88 \\
979,913.85 \\
980,052.96 \\
, 045.88\end{array}$ \\
\hline & $\begin{array}{l}\text { Errore di } \\
\text { chiusura: }\end{array}$ & -0.11 & 0.00 & \\
\hline \multirow[t]{2}{*}{ XIII } & $\begin{array}{l}\text { Catania } \\
\text { Messina } \\
\text { S. Stefano di } \\
\text { Camastra } \\
\text { Catania }\end{array}$ & $\begin{array}{l}+\quad 59.07 \\
-\quad 57.30 \\
-\quad 1.45\end{array}$ & $\begin{array}{r}+58.97 \\
-\quad 57.46 \\
-\quad 1.51\end{array}$ & $\begin{array}{r}980,045.88 \\
.104 .85 \\
, 047.39 \\
, 045.88\end{array}$ \\
\hline & $\begin{array}{l}\text { Errore di } \\
\text { chiusura: }\end{array}$ & $+\quad 0.32$ & 0.00 & \\
\hline
\end{tabular}


Applicando le correzioni $x_{i}$ sopra ricavate, le differenze di gravità compensate, per i lati della rete di $1^{\circ}$ ordine vengono ad assumere $i$ valori indicati nella Tabella $I$, e gli errori di chiusura di tutti i triangoli e del poligono completo diventano nulli: ciò comprova che la compensazione eseguita è sufficiente.

La precisione " esterna " dei valori della gravità nei vertici del $1^{0}$ ordine resta praticamente quella dei caposaldi fondamentali, Palermo e Catania $\left({ }^{3}\right)$, nel sistema di Potsdam: cioè, +0.4 mgal.

Le stazioni di riferimento di $1^{\circ}$ ordine sono riportate in grassetto nella Tabella IIT, e pure in grassetto sono riportati nella Tabella IV i valori compensati di $g$ che ad esse competono.

3. Rete di $2^{\circ}$ ordine. - Per le necessità dei collegamenti di dettaglio, è stala istituila una rete di stazioni di riferimento di $2^{\circ}$ ordine, distribuite e collegate con quelle di $1^{\circ}$ ordine come indicato dalla fig. I, e con i valori riportati nella Tabella II. Essendo esse distribuite in poligoni chiusi, e data l'esiguità degli errori di chiusura, che risultano dalla Tabella II, è stata effettuata una compensazione speditiva, lasciando inalterati $i$ valori delle stazioni $d i 1^{0}$ ordine risultanti dalla compensazione di cui al $\$ 2$, e distribuendo proporzionalmente gli errori di chiusura, secondo quanto indicato nella Tabella 1I. La precisione " interna " di queste stazioni si può accettare senz'altro dell'ordine di +0.2 mgal.

Le stazioni di riferimento di $2^{\circ}$ ordine sono riportate in corsivo nella Tabella III, e pure in corsivo sono riportati nella Tabella $\mathrm{V}$ i valori compensati di $g$ che ad esse competono.

4. Punti di dettaglio. - Secondo le raccomandazioni dell'Unione Geodetica e Geofisica Internazionale, accettate per l'Italia dalla Commissione Geodetica Italiana, la densità media delle stazioni gravimetriche per i grandi rilievi nazionali dovrebbe essere di almeno una ogni $100 \mathrm{~km}^{2}$. Tale rapporto comportereblue per la Sicilia 257 stazioni; il rilievo di cui qui si rende conto consta invece di 299 stazioni. Esse sono riportate in ordine alfabetico nella Tabella III, con la posizione riferita ai f. 100.000 dell'I.G.M., il riferimento altimetrico e la descrizione (per quasi tutte le stazioni esiste anche la fotografia).

La distribuzione delle stazioni risulta dalla fig. 2 , in cui sono riportati anche $i$ collegamenti per le stazioni di dettaglio. Come si vede tale distribuzione ̀̀ uniforme in maniera soddisfacente, se si pensa in particolare al carattere montuoso e impervio di tutta la re- 


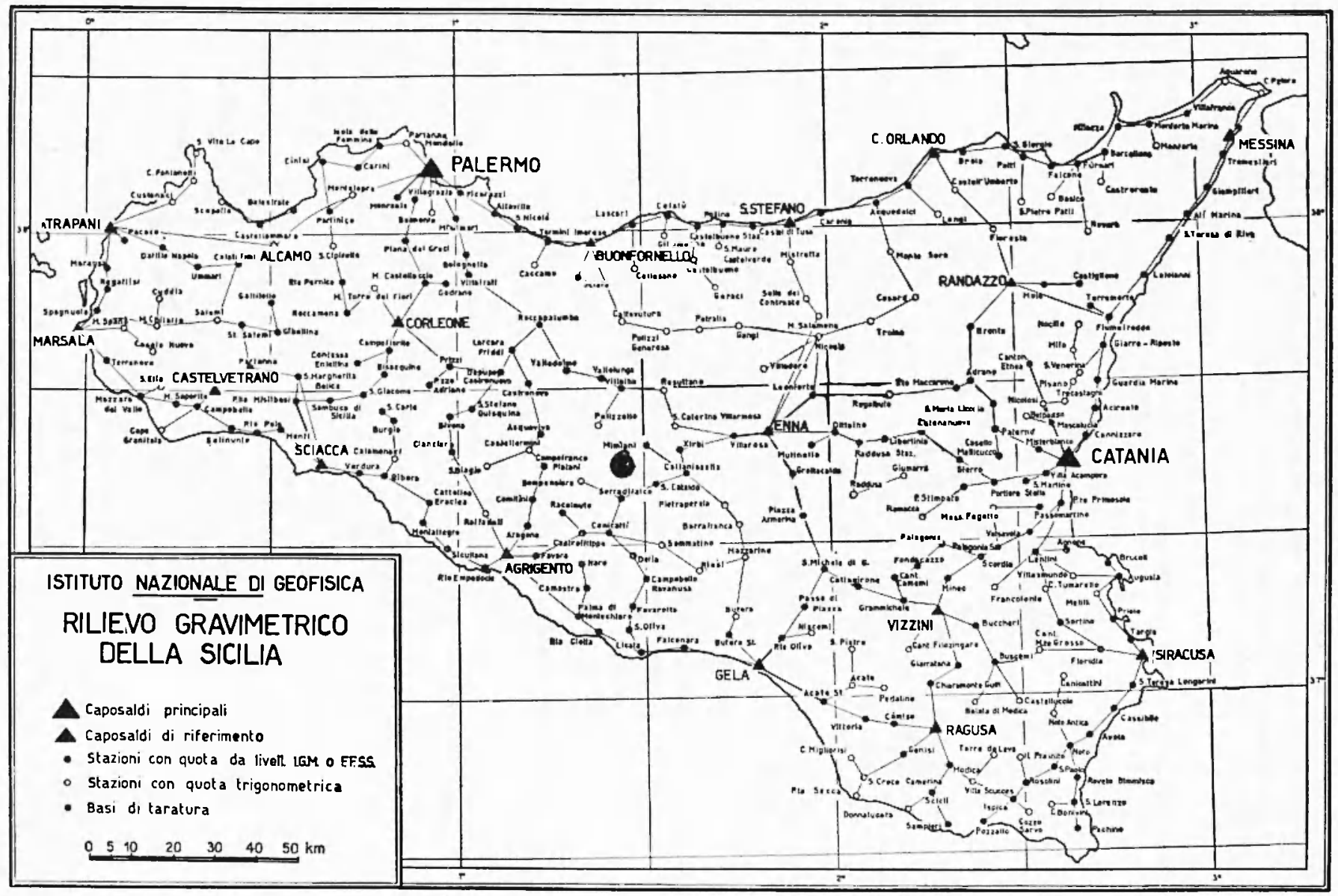

Fig. 2 - Stazioni gravimetriche di dettaglio e schema dei collegamenti 


\section{Tabella III}

Descrizione delle stazioni gravimetriche per ordine alfabetico, posizione nei fogli al 100.000 e riferimenti altimetrici.

\begin{tabular}{|c|c|c|c|c|}
\hline Stazione & $\mathbf{F} / \mathbf{q} / \mathbf{t}$ & Descrizione & $\begin{array}{l}\text { Riferimento } \\
\text { altimetrico }\end{array}$ & N. \\
\hline Acate & 273.III.SW & $\begin{array}{l}\text { Castello Principe di Paterno: lato } \mathrm{W} \\
\text { torre sull'angolo SW }\end{array}$ & p.to trigon. & 266 \\
\hline Acate Staz. & 275.I.NE & Stazione ff.ss. $(*)$ & p.d.f. & 270 \\
\hline Acireale & 270.IV.NE & Stazione ff.ss. & cs.v. IGM & 152 \\
\hline Acquaviva & 267.IV.SE & Stazione ff.ss. & p.d.f. & 162 \\
\hline Acquedolci & 252.1II.SE & $\begin{array}{l}\text { Al centro del muro sito ad Est dell'edi- } \\
\text { ficio della stazione ff.ss. }\end{array}$ & p.d.f. & 35 \\
\hline Adrano & 261.II.SE & Stazione Circumetnea & p.d.f. & 133 \\
\hline Agnone & 274.IV.NE & Stazione ff.ss. & p.d.f. & 221 \\
\hline $\begin{array}{l}\text { Agrigento } \\
\text { Alcamo }\end{array}$ & 271.JV.NE & Stazione ff.ss. (bassa) & p.d.f. & 216 \\
\hline Alcamo & 258.IV.NW & $\begin{array}{l}\text { Presso il marciapiedi, davanti all'entra- } \\
\text { ta principale della Chiesa Mad. Soc- } \\
\text { corso }\end{array}$ & p.to trigon. & 59 \\
\hline Ali Marina & 255.II.SE & $\begin{array}{l}\text { Ai piedi del cs.v. IGM posto a destra } \\
\text { del portone principale dell'Istituto } \\
\text { Magistrale femm. "Maria Ausiliatri- } \\
\text { ce" }\end{array}$ & cs.v. IGMI & 52 \\
\hline Altavilla & $250.11 I . S W$ & Stazione ff.ss. & cs.v. IGMI & 39 \\
\hline Aquarone & 254.IV.NW & $\begin{array}{l}\text { Al } \mathrm{km} .19,9 \text { della strada costiera, da- } \\
\text { vanti al cancello della villa sita sulla } \\
\text { riva destra del fiume Casagrande (a } \\
\text { monte della strada) }\end{array}$ & p.to quotato & 1 \\
\hline Aragona & 267.III.SE & Stazione ff. Aragona-Caldare & p.d.f. & 208 \\
\hline Augusta & 274.I.SW & Stazione ff.ss. & p.d.f. & 235 \\
\hline Avola & 277.IV.SE & Stazione ff.ss. & p.d.f. & 278 \\
\hline Balata di Modica & 276.I.NW & $\begin{array}{l}\text { Case Migliorisi: a } 40 \text { no dalla strada } \\
\text { Giarratana-Modica presso il bivio di } \\
\text { S. Giacomo, in mezzo allo spiazzo }\end{array}$ & p.to quotato & 272 \\
\hline Balestrate & 249.III.SW & Stazione ff.ss. & p.d.f. & 36 \\
\hline Barcellona & 253.II.NW & $\begin{array}{l}\text { Davanti allo stipite destro del cancello } \\
\text { di ingresso allo scalo merci della sta- } \\
\text { zione ff.ss. }\end{array}$ & p.d.f. & 15 \\
\hline Barrafranca & 268.III.SE & $\begin{array}{l}\text { Chiesa Madre: alla base del campanile, } \\
\text { sulla via V. Em. }\end{array}$ & p.to trigon. & 209 \\
\hline Basico & 253.III.SW & $\begin{array}{l}\text { Al centro della facciata della Chiesa } \\
\text { Madre }\end{array}$ & p.to trigon. & 33 \\
\hline Belmonte & 241.JI.SE & $\begin{array}{l}\text { Sul piazzale della chiesa allinizio della } \\
\text { scalinata }\end{array}$ & p.to trigon. & 37 \\
\hline Belpasso & 270.IV.NW & $\begin{array}{l}\text { Al centro della facciata della Chiesa del } \\
\text { Purgatorio, adiacente al Municipio }\end{array}$ & p.to quotato & 156 \\
\hline Bisacquino & 258.II.SW & $\begin{array}{l}\text { Sulla piazza, all'incrocio degli assi del } \\
\text { municipio e del campanile }\end{array}$ & cs.v. IGMI & 122 \\
\hline $\begin{array}{l}\text { Bivona } \\
\text { Bolognetta }\end{array}$ & $\begin{array}{l}\text { 266.I.NE } \\
\text { 259.IV.NW }\end{array}$ & $\begin{array}{l}\text { Stazione ff.ss. } \\
\text { A } 4 \mathrm{~m} \text { a } \mathrm{S} \text { dallo spigolo } \mathrm{SW} \text { del fab- } \\
\text { bricato della stazione ft.ss. }\end{array}$ & $\begin{array}{l}\text { p.d.f. } \\
\text { p.d.f. }\end{array}$ & $\begin{array}{r}151 \\
63\end{array}$ \\
\hline
\end{tabular}

(*) Con questa abbreviazione si intenderà, qui e nel seguito: " $\Delta$ l centro della facciata della stazione ferroviaria 》. 
.. segue Tab. III

\begin{tabular}{|c|c|c|c|c|}
\hline Stazione & $\mathbf{F} / \mathrm{q} / \mathrm{t}$ & Descrizione & $\begin{array}{l}\text { Riferimento } \\
\text { altimetrico }\end{array}$ & N. \\
\hline Bomprensiere & 267.II.NW & Al centro della facciata della Chiesa & p.to quotato & 188 \\
\hline Brolo & 252.II.NE & Stazione ff.ss. & p.d.f. & II \\
\hline Bronte & 261.II.NE & $\begin{array}{l}\text { Davanti la facciata } S \text { magazzino scalo } \\
\text { merci }\end{array}$ & p.d.f. & 106 \\
\hline Brucoli & 274.IV.NE & Stazione fi.ss. & p.d.f. & 229 \\
\hline Buccheri & 273.II.NE & Stazione ff.ss. & p.d.f. & 249 \\
\hline Buonfornello & 259.I.NW & $\begin{array}{l}\text { Davanti alla piccola costruzione (pol- } \\
\text { laio) aggiunta a destra della stazio- } \\
\text { ne fi.ss. }\end{array}$ & p.d.f. & 62 \\
\hline Burgio & 266.I.NW & Starione ff.ss. & p.d.f. & 153 \\
\hline Buscemi & 273.II.SE & Stazione ff.ss. & p.d.f. & 264 \\
\hline Butera & 272.IV.SE & Strada Gelo-Butera: curva al $\mathrm{km} 13,8$ & p.to quotato & 245 \\
\hline Butera Staz. & 272.III.NE & Stazione ff.ss. & p.d.f. & 252 \\
\hline Caccamo & 259.IV.NE & $\begin{array}{l}\text { Davanti al capitello fra il } \mathrm{km} 9 \text { e } 10 \\
\text { sulla strada provinciale Termini-Cac. } \\
\text { camo }\end{array}$ & p.to quotato & 67 \\
\hline Calamonaci & 266.I.SW & $\begin{array}{l}\text { Al centro della facciata della Chiesa di } \\
\text { S. Vincenzo }\end{array}$ & p.to trigon. & 176 \\
\hline Calatafimi & 257.I.NE & Stazione ff.ss. & p.d.f. & 68 \\
\hline Caltagirone & 273.IV.SW & Stazione ff.ss. & cs.v. IGMI & 237 \\
\hline Calt anissetta & 268.III.NW & Stazione ff.ss. & p.d.f. & 185 \\
\hline Caltavuturo & 259.II.NE & $\begin{array}{l}\text { Sulla statale, al bivio per Valledolmo, } \\
4 \mathrm{~m} \text {. prima dal cippo d'origine }\end{array}$ & p.to quotato & 95 \\
\hline Camastra & 271.I.SW & Stazione ff.ss. & p.d.f. & 232 \\
\hline Camemi & 273.IV.NE & $\begin{array}{l}\text { Al centro della facciata della cantonie- } \\
\text { ra provinciale sulla strada Caltagiro- } \\
\text { ne-Catania }\end{array}$ & cs.v. IGM & 231 \\
\hline Campobello & 265.I.NW & $\begin{array}{l}\text { Davanti all'ingresso della Chiesa S. Mi- } \\
\text { chele }\end{array}$ & cs.v. IGM & 145 \\
\hline $\begin{array}{l}\text { Campobello- } \\
\text { Ravanusa }\end{array}$ & 271.I.NE & Stazione ff.ss. & df & 930 \\
\hline Campofiorito & 258.II.NW & $\begin{array}{l}\text { Davanti al cs.v. a destra del portone } \\
\text { della caserma CC. }\end{array}$ & cs.v. IGM & 112 \\
\hline Campofranco & 267.III.NE & Stazione fi.ss. & p.d.f. & 180 \\
\hline C'anicattì & 267.II.SE & Stazione ff.ss. & p.d.f. & 211 \\
\hline Canicattini & 274.III.SW & $\begin{array}{l}\text { Davanti alla porta d'ingresso Chiesa del } \\
\text { Purgatorio, in via dei Mille }\end{array}$ & p.to trigon. & 265 \\
\hline Cannizzaro & 270.IV.SE & $\begin{array}{l}\text { Stazione ff.ss.: al centro della facciata } \\
\text { Est del magazzino scalo merci }\end{array}$ & p.d.f. & 170 \\
\hline Cant. Etna (est.) & 262.III.SW & $\begin{array}{l}\text { Al centro del piazzale, a } 7 \text { m dall'ini- } \\
\text { zio della strada per il rifugio Sapienza }\end{array}$ & p.to quotato & 123 \\
\hline Cant. Filozingaro & 273.III.NE & $\begin{array}{l}\text { Al centro della facciata della cantonie- } \\
\text { ra fra i km } 19 \text { e } 20 \text { della strada } \mathrm{Li} \text { - } \\
\text { codia-Vittoria }\end{array}$ & p.to quotato & 258 \\
\hline Cant. Monte Grosso & 274.III.NW & $\begin{array}{l}\text { Al } \mathrm{km} 90+992 \text { della S.S. } 124: \text { al cen- } \\
\text { tro della facciata }\end{array}$ & p.to quotato & 259 \\
\hline Capo Granitola & 265.IV.SE & Davanti al cancello d'ingresso al faro & l.m.m. & 165 \\
\hline Capo Orlando & 252.II.NW & $\begin{array}{l}\text { Stazione ff.ss.: } 3 \mathrm{~m} \text { a sinistra dello sti- } \\
\text { pite sinistro del cancello d'ingresso } \\
\text { scalo merci }\end{array}$ & p.d.f. & 12 \\
\hline
\end{tabular}


... eegue Tab. IlI

\begin{tabular}{|c|c|c|c|c|}
\hline Stazione & $\mathbf{F} / \mathbf{q} / \mathrm{t}$ & Descrizione & $\begin{array}{l}\text { Riforimento } \\
\text { altimetrico }\end{array}$ & N. \\
\hline Capo Peloro & 254.IV.NE & Davanti al cancello d'ingresso al Faro & p.to trigon. & 2 \\
\hline Carini & 249.III.NE & Stazione ff.ss. & p.d.f. & 16 \\
\hline Caronia & 251.II.SE & Stazione ff.ss. & p.d.f. & 40 \\
\hline Casale Nuovo & 257.III.NE & $\begin{array}{l}\text { Davanti al portone a volta n. } 954 \text {, sotto } \\
\text { il poggiolo }\end{array}$ & p.to quotato & 111 \\
\hline Casa Tumarello & 274.IV.SW & $\begin{array}{l}\text { Davanti al volto d'ingresso, sulla stra- } \\
\text { da Lentini-Sortino }\end{array}$ & p.to quotato & 238 \\
\hline Case Bonivini & 277.III.NW & $\begin{array}{l}\text { Strada Rosolini-Pachino, all'inizio del- } \\
\text { la mulattiera per dette case }\end{array}$ & p.to quotato & 294 \\
\hline Case Fontanotti & 248.II.NW & $\begin{array}{l}\text { Sulla strada Custonaci-S. Vito Lo Capo, } \\
\text { al bivio per Case Fontanotti }\end{array}$ & p.to quotato & 20 \\
\hline Casello Mellicucco & 269.I.SE & $\begin{array}{l}\text { Strada Paternò-Staz. Simeto, contrada } \\
\text { Ficuzza: davanti alla porta d'ingresso }\end{array}$ & cs.v. IGM & 178 \\
\hline Case Migliorisi & 276.IV.SW & $\begin{array}{l}\text { Davanti alla porta sulla facciata } \mathrm{N} \text { della } \\
\text { casa avente presso lo spigolo } \mathrm{NE} \text { un } \\
\text { pozzo }\end{array}$ & p.to trigon. & 281 \\
\hline Cassibile & 277.IV.NE & Stazione ff.ss. & p.d.f. & 274 \\
\hline Castelbuono & 260.IV.NE & $\begin{array}{l}\text { All'origine e sull'asse della strada per } \\
\text { il cimitero, sul ciglio della provin- } \\
\text { ciale }\end{array}$ & p.to quotato & 69 \\
\hline Castelbuono Staz. & 251.III.SE & Stazione ff.ss. & p.d.f. & 49 \\
\hline Castel di Tusa & 251.II. SW & Stazione ff.ss. & p.d.f. & $\begin{array}{l}51 \\
41\end{array}$ \\
\hline $\begin{array}{l}\text { Castellammare } \\
\text { Castelluccio }\end{array}$ & $\begin{array}{l}\text { 248.II.SE } \\
\text { 276.I.NE }\end{array}$ & $\begin{array}{l}\text { Stazione ff.ss. } \\
\text { Strada prov. Palazzolo Acreide - Noto, } \\
\text { in mezzo al bivio per Castelluccio }\end{array}$ & $\begin{array}{l}\text { p.d.f. } \\
\text { p.to quotato }\end{array}$ & $\begin{array}{r}41 \\
271\end{array}$ \\
\hline Castell'Umberto & 252.II.NW & $\begin{array}{c}\text { Davanti al lato } \mathrm{N} \text { del campanile della } \\
\text { chiesa }\end{array}$ & p.to trigon. & 27 \\
\hline Casteltermini & 267.IV.SE & $\begin{array}{l}\text { Sulla piazza S. Filippo, di fronte all'in- } \\
\text { gresso laterale della Chiesa Madre, } \\
\text { presso il marciapiede }\end{array}$ & p.to trigon. & 172 \\
\hline Castelvetrano & 257.II.SW & $\begin{array}{l}\text { Davanti alla scalinata della Chiesa della } \\
\text { Immacolata }\end{array}$ & cs. o IGM & 131 \\
\hline Castiglione & 262.IV.SE & Stazione ff.ss. & p.d.f. & $\begin{array}{r}82 \\
210\end{array}$ \\
\hline Castrofilippo & 267.II.SW & Stazione ff.ss. & p.d.f. & 210 \\
\hline Castronovo & 259.JII.SE & Stazione ff.ss. & p.d.f. & 129 \\
\hline Castroreale & 253.1II.NE & $\begin{array}{l}\text { Al centro della facciata della casa più } \\
\text { alta in contrada Casabianca, sulla stra- } \\
\text { da che conduce a Bafia }\end{array}$ & p.to quotato & 26 \\
\hline CATANIA & 270.IV.SE & $\begin{array}{l}\text { Osservatorio Astrofisico sul piazzale } \\
\text { Vaccarini davanti al cs.v. IGM, ad } 1 \\
\text { mal 10 scalino }\end{array}$ & cs.v. IGM & 182 \\
\hline Catenanuova & 269IV.SE & Stazione ff.ss. & p.d & 167 \\
\hline Cattolica Eraclea & 266.II.NE & Stazione ff.ss. & p.d.f. & 196 \\
\hline $\begin{array}{l}\text { Cefalù } \\
\text { Cesarò }\end{array}$ & $\begin{array}{l}\text { 251.III.SW } \\
\text { 261.IV.SE }\end{array}$ & $\begin{array}{l}\text { Stazione ff.ss. } \\
\text { Sulla strada Cesarò - S. Fratello al bivio }\end{array}$ & $\begin{array}{l}\text { p.d.f. } \\
\text { p.to quotato }\end{array}$ & $\begin{array}{l}42 \\
87\end{array}$ \\
\hline Chiaramonte-Gulfi & 273.II.SW & $\begin{array}{l}\text { per S. Teodoro } \\
\text { Davanti al cancelletto presso lo spigolo } \\
\text { NW della stazione ff.ss. }\end{array}$ & p.d.f. & 268 \\
\hline Cianci & 266.I.SE & Stazione ff.ss. & p.d & 175 \\
\hline Cinisi & 249.III.NE & Stazione ff.ss. & p.d.f. & 14 \\
\hline
\end{tabular}


... segue Tab. III

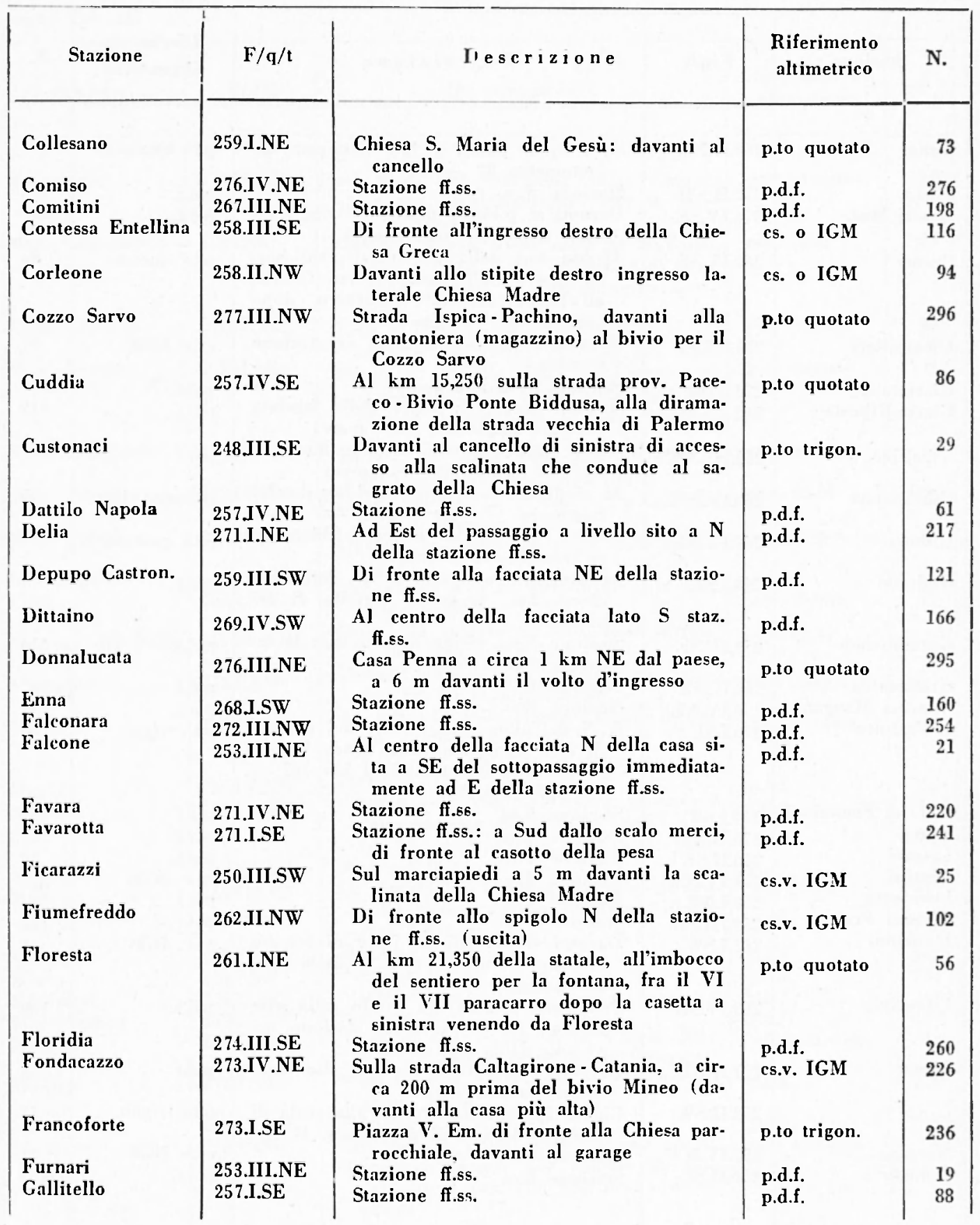


... segue Tab. III

\begin{tabular}{|c|c|c|c|c|}
\hline Stazione & $\mathrm{F} / \mathbf{q} / \mathrm{t}$ & Deserizione & $\begin{array}{l}\text { Riferimento } \\
\text { altimetrico }\end{array}$ & $\mathbf{N}$. \\
\hline Gangi & 260.II.NW & $\begin{array}{l}\text { Sulla strada statale n. } 120 \text {, dirimpetto al- } \\
\text { l'ettometro } 82+\text { IV }\end{array}$ & p.to quotato & 101 \\
\hline Gela & 272.II.SW & Stazione ff.ss. & p.d.f. & 261 \\
\hline Genisi Staz. & 276.IV.SE & $\begin{array}{l}\text { Davanti al pilastro destro }(\mathrm{N}) \text { del can- } \\
\text { cello d'ingresso scalo merci }\end{array}$ & p.d.f. & 280 \\
\hline Ceraci & 260.IV.SE & $\begin{array}{l}\text { Al } \mathrm{km} 33,4 \text { della provinciale, sul bor- } \\
\text { do destro della strada verso Geraci, } \\
\text { all'altezza del } 12^{\circ} \text { paracarro dopo } \\
\text { l'origine del sentiero }\end{array}$ & p.to quotato & 83.3 \\
\hline Giampilieri & 254.III.SW & $\begin{array}{l}\text { Davanti al cs.v. IGM sulla ex stazione } \\
\text { tramviaria }\end{array}$ & cs.v. IGM & 32 \\
\hline Giarratana & 273.II.SW & Stazione ff.ss., lato sud & p.d.f. & 263 \\
\hline Giarre-Riposto & 262.III.SE & $\begin{array}{l}\text { Stazione ff.ss.: al centro della facciata } \\
\text { NW del magazzino scalo merci }\end{array}$ & p.d.f. & 115 \\
\hline Gibellina & 258.III.NW & $\begin{array}{l}\text { presso spigolo sinistra stazione ff.ss., da- } \\
\text { vanti al cancelletto d'ingresso }\end{array}$ & p.d.f. & 107 \\
\hline Gibilmanna & 260.IV.NW & $\begin{array}{l}\text { Ai piedi del campanile meridionale del } \\
\text { Santuario }\end{array}$ & pozzetto bar. & 55 \\
\hline Giumarra & 269.III.NE & $\begin{array}{l}\text { Davanti al cancello di casa Chianotta, } \\
\text { in fraz. Castel di ludica }\end{array}$ & p.to quotato & 189 \\
\hline Godrano & 258.I.SE & $\begin{array}{l}\text { Al passaggio a livello a } \mathrm{SW} \text { della Sta- } \\
\text { zione f.ss., presso il cartello di av- } \\
\text { viso a } \mathrm{S}\end{array}$ & p.d.f. & 78 \\
\hline Grammichele & 273.IV.SE & $\begin{array}{l}\text { Stazione ff.ss., vicino al palo con la sa- } \\
\text { goma altezza carri }\end{array}$ & p.d.f. & 240 \\
\hline Grottacalda & 268.II.NF & Stazione ff.ss. & p.d.f. & 187 \\
\hline Il Prainito & 276.I.SE & $\begin{array}{l}\text { A N dellalbero del cortile del gruppo } \\
\text { di case omonimo sulla strada Ritilli- } \\
\text { ni - Modica dipartentesi al km } 365+ \\
\text { III dalla S.S. } 115\end{array}$ & $\begin{array}{l}\text { p.d.t. } \\
\text { p.to trigon. }\end{array}$ & $\begin{array}{l}135 \\
282\end{array}$ \\
\hline Isola d. Femmine & 24.9.I.SW & Stazione ff.ss. & p.d.f. & 7 \\
\hline Ispica. & 276.II.NE & Stazione ff.ss. & p.d.f. & 242 \\
\hline Lascari & 250.II.SE & Staxione ff.ss. & p.d.f. & 50 \\
\hline Lentini & 274.IV.NW & Stazione ff.ss. & es.v. IGM & 222 \\
\hline Leonforte & 268.I.NE & Stazione fi.ss. & p.d.f. & 143 \\
\hline $\begin{array}{l}\text { Lercara Friddi } \\
\text { Letoianni }\end{array}$ & $\begin{array}{l}\text { 259.III.SE } \\
\text { 262.I.SW }\end{array}$ & $\begin{array}{l}\text { Stazione ff.se. } \\
\text { Davanti al cance!lo in ferro di accesso } \\
\text { al palazzo Durante, al n. } 18 \text { della piaz. } \\
\text { za omonima }\end{array}$ & $\begin{array}{l}\text { p.d.f. } \\
\text { cs.v. IGM }\end{array}$ & $\begin{array}{r}114 \\
81\end{array}$ \\
\hline Libertinia & 269.IV.SE & $\begin{array}{l}\text { Prima del passaggio a livello sulla stra- } \\
\text { dicciola che conduce alla stazione, de- } \\
\text { viando dalla provinciale }\end{array}$ & p.d.f. & 169 \\
\hline Licata & 271.II.NE & $\begin{array}{l}\text { Davanti a cancello d'ingresso allo scalo } \\
\text { merci stazione ff.ss. }\end{array}$ & p.d.f. & 256 \\
\hline Longi & 252.II.SW & $\begin{array}{l}\text { Chiesa Madre: dirimpetto alla scala di } \\
\text { sinistra, davanti al portone n. } 41\end{array}$ & p.to trigon. & 43 \\
\hline $\begin{array}{l}\text { Marausa } \\
\text { Marsala }\end{array}$ & $\begin{array}{l}\text { 257.IV.NW } \\
\text { 256.II.SE }\end{array}$ & $\begin{array}{l}\text { Stazione ff.ss. } \\
\text { Stazione ff.ss. }\end{array}$ & $\begin{array}{l}\text { es.v. IGM } \\
\text { p.d.f. }\end{array}$ & $\begin{array}{r}72 \\
103\end{array}$ \\
\hline
\end{tabular}


... segue Tab. III

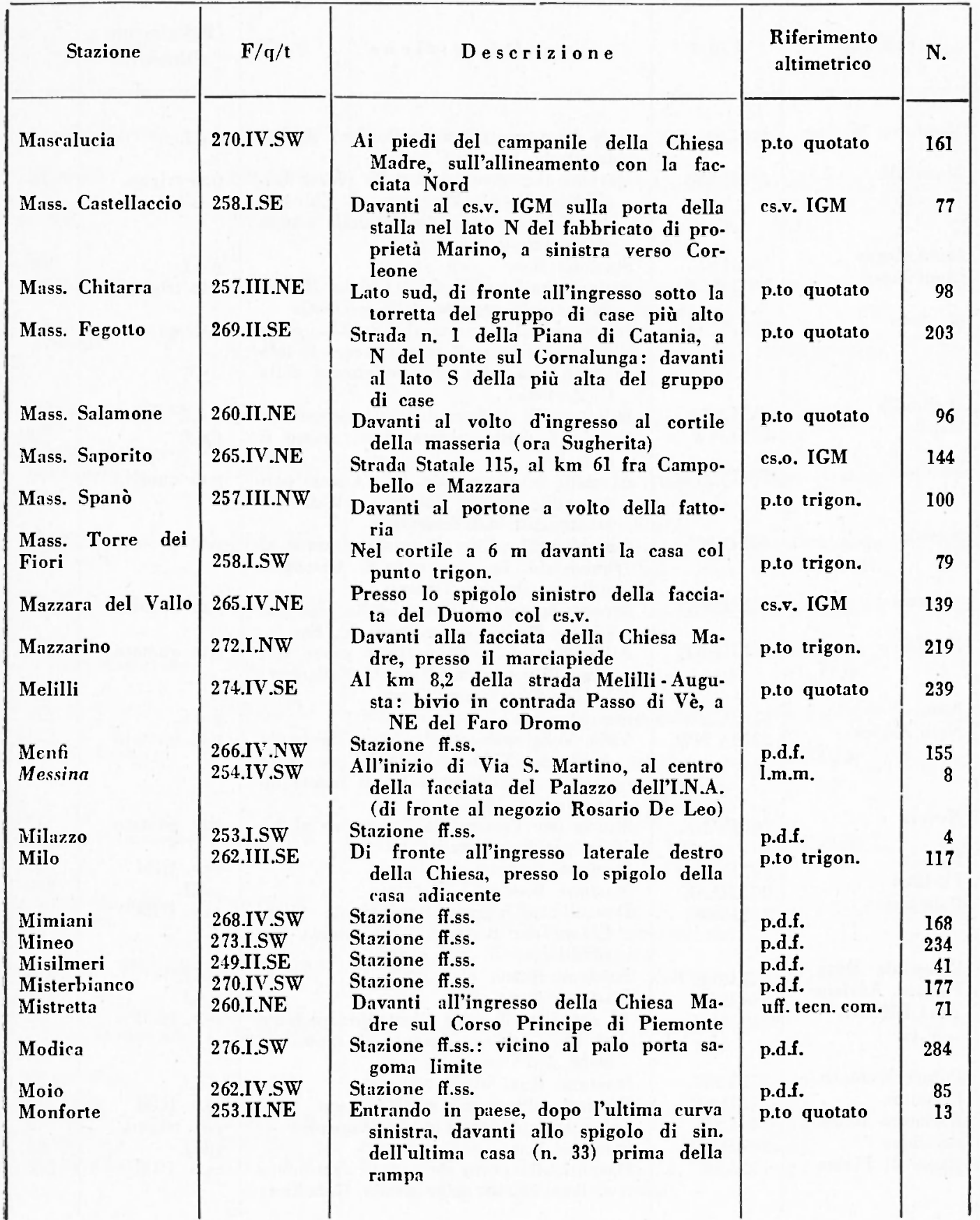


... segue Tab. III

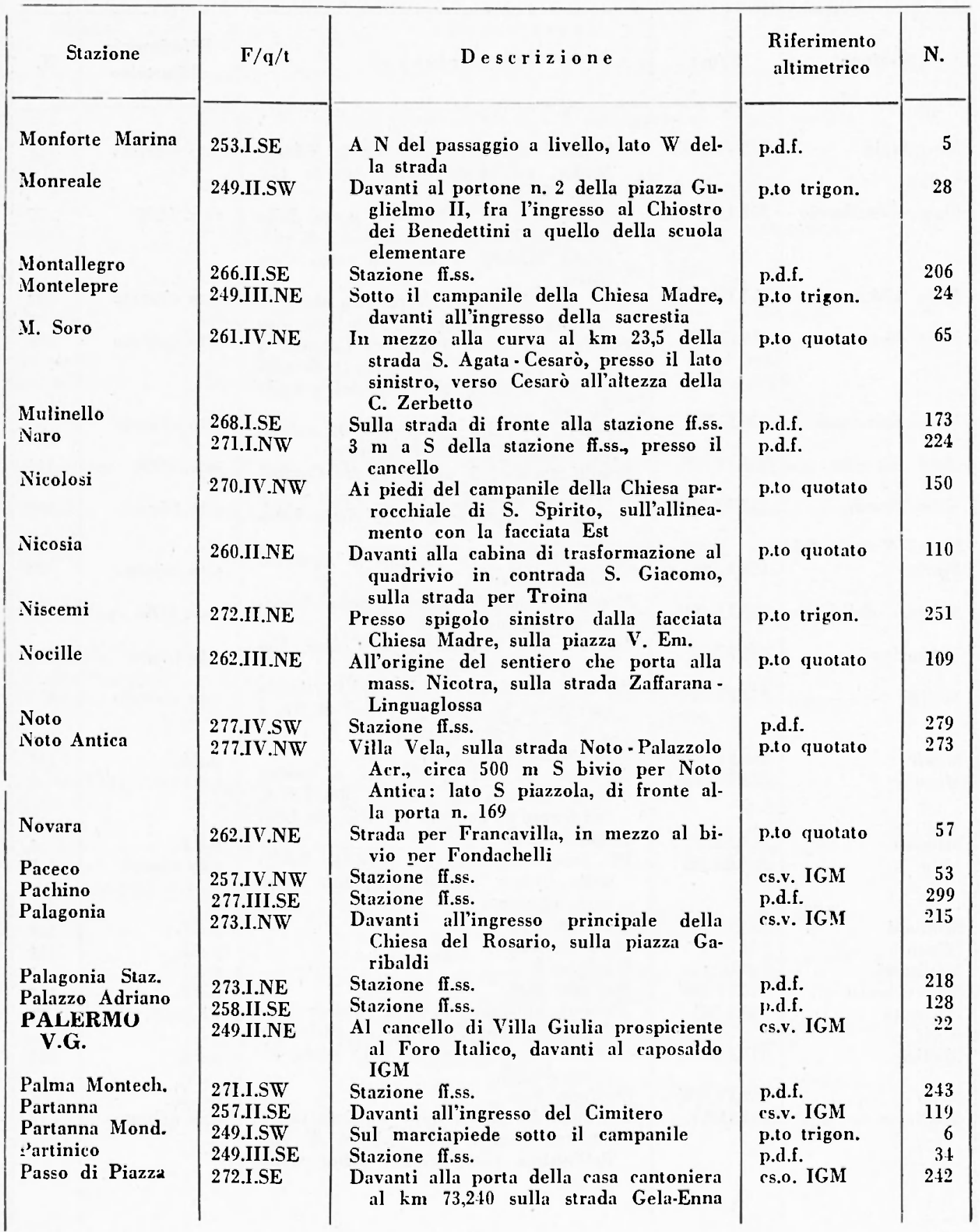


... segue Tab. III

\begin{tabular}{|c|c|c|c|c|}
\hline Stazione & $\mathbf{F} / \mathrm{q} / \mathrm{t}$ & Descrizione & $\begin{array}{l}\text { Riferimento } \\
\text { altimetrico }\end{array}$ & N. \\
\hline Passo Martino & 270.III.SW & $\begin{array}{l}\text { Case dei ferrovieri: al centro della fac- } \\
\text { ciata W della casa meridionale }\end{array}$ & p.d.f. & 204 \\
\hline Paterno & 269.I.SE & $\begin{array}{l}\text { Davanti alla porta destra della Chiesa } \\
\text { di S. Barbara }\end{array}$ & cs.v. IGM & 164 \\
\hline Patti & 253.III.NW & $\begin{array}{l}\text { Davanti allo stipite destro dal cancello } \\
\text { d'ingresso allo scalo merci della sta- } \\
\text { yione ff.ss. }\end{array}$ & p.d.f. & 17 \\
\hline Pedalino & 273.III.SW & $\begin{array}{l}\text { Davanti alla prima casa a sinistra (n. } \\
\text { 388), entrando in paese da W, fra } \\
\text { la porta e la finestra }\end{array}$ & p.to trigon. & 267 \\
\hline Petralia & 260.III.NE & $\begin{array}{l}\text { Sulla strada statale n. } 120 \text { al bivio Ali- } \\
\text { mena-Carda, davanti alla pietra mi- } \\
\text { liare }\end{array}$ & p.to quotato & 105 \\
\hline Piana dei Greci & 258.I.NW & $\begin{array}{l}\text { Davanti al km } 27 \text { sulla strada Piana } \\
\text { dei Greci-Bivio Lupotto }\end{array}$ & cs.o. IGM & 58 \\
\hline Piazza Armerina & 268.II.SE & $\begin{array}{l}\text { de1 Grec1- BIv1o Lupotto } \\
\text { Stazione ff.ss. }\end{array}$ & p.d.f. & 205 \\
\hline Pietraperzia & 268.III.NE & $\begin{array}{l}\text { Sulla strada Varco Ramata - Pietraper- } \\
\text { zia, di fronte all'ingresso casa canto- } \\
\text { niera al bivio per Caltanissetta }\end{array}$ & p.to quotato & 199 \\
\hline Pisano & 270IV.NE & Al centro della facciata della chiesa & p.to quotato & 134 \\
\hline Polizzello & 267.I.NE & $\begin{array}{l}5 \mathrm{~m} \text { a Sud km } 23 \text { strada Villalba-Mus- } \\
\text { someli }\end{array}$ & p.to quotato & 154 \\
\hline Polizzi Generosa & 260.JII.NW & $\begin{array}{l}\text { Sulla S.S. 120, in mezzo al bivio per } \\
\text { Polizzi Generosa }\end{array}$ & p.to quotato & 108 \\
\hline Pollina & 251.III.SE & Stazione ff.ss. & p.d.f. & 45 \\
\hline Ponte Maccarone & 269.I.NW & $\begin{array}{l}\text { Davanti al cs.v. sulla casa sita al bivio } \\
\text { per Paternó, S.S. } 121\end{array}$ & cs.v. IGM & 138 \\
\hline Ponte Olivo & 272.II.NW & $\begin{array}{l}\text { Sulla strada Gela - Enna, estremità spal- } \\
\text { letta a monte riva destra }\end{array}$ & cs.v. IGM & 253 \\
\hline Ponte Pernice & 258.IV.SE & $\begin{array}{l}\text { Al bivio per Camporeale sulla strada } \\
\text { da S. Cipirello a Roccamena, davanti } \\
\text { al cs.v. IGM sullo spigolo NE casa } \\
\text { Ponte Pernice }\end{array}$ & cs.v. IGM & 76 \\
\hline Ponte Primosole & 270.III.SW & $\begin{array}{l}\text { Sulla S.S. n. } 114 \text {, al } \mathrm{km} 111+\text { VII, da- } \\
\text { vanti al cancello d'ingresso della mas- } \\
\text { seria Di Stefano }\end{array}$ & cs.o. IGM & 202 \\
\hline Ponte Stimpato & 269.II.NW & $\begin{array}{l}\text { Sulla strada da Catania, a } 30 \mathrm{~m} \text { passa- } \\
\text { to il ponte, allinizio del sentiero che }\end{array}$ & cs.o. IGM & 195 \\
\hline Portella Misilbesi & 266.IV.NW & $\begin{array}{l}\text { porta alla Masseria Stimpato } \\
\text { Davanti alla porta di destra dell'avan- } \\
\text { corpo della ex caserma CC. }\end{array}$ & cs.o. IGM & 140 \\
\hline Portiere Stella & 269.II.NE & Stazione ff.ss. lato binari & p.d.f. & 193 \\
\hline Porto Empedocle & 271.IV.NW & $\begin{array}{l}\text { Stazione ff.ss.: fra la fontanella in mez- } \\
\text { zo all'aiuola ed i binari }\end{array}$ & p.d.f. & 225 \\
\hline $\begin{array}{l}\text { Porto Palo } \\
\text { Pozzallo }\end{array}$ & $\begin{array}{l}\text { 265.I.SE } \\
276 \text { II SF }\end{array}$ & Stazione ff.ss. & p.d.f. & 159 \\
\hline Priolo & 27.1.III.NE & $\begin{array}{ll}\text { Stazione } & \text { If.ss. } \\
\text { Stazione ff.ss. }\end{array}$ & $\begin{array}{l}\text { p.o.r. } \\
\text { p.d.f. }\end{array}$ & $\begin{array}{l}291 \\
246\end{array}$ \\
\hline Prizzi & 258.II.SE & Stazione ff.ss. & p.d.f. & 118 \\
\hline Punta Ciotta & 271.II.NW & $\begin{array}{l}\text { Passaggio a livello al } \mathrm{km} 46.754 \text { linea } \\
\text { Licata-Palma, presso il casello }\end{array}$ & p.d.f. & 2.47 \\
\hline
\end{tabular}


... segue Tab, IlI

\begin{tabular}{|c|c|c|c|c|}
\hline Stazione & $\mathbf{F} / \mathbf{q} / \mathrm{t}$ & Descriaione & $\begin{array}{l}\text { Riferimento } \\
\text { altimetrico }\end{array}$ & N. \\
\hline Punta Secca & 276.III.NW & $\begin{array}{l}\text { Davanti al cancello d'ingresso al recin- } \\
\text { to del faro }\end{array}$ & p.to trigon. & 291 \\
\hline Racalmuto & 267.II.SW & Stazione ff.ss. & p.d.f. & 200 \\
\hline Raddusa & 269.III.NW & $\begin{array}{l}\text { Strada Raddusa - Caltagirone in mezzo } \\
\text { al bivio per Aidone }\end{array}$ & p.to quotato & 194 \\
\hline $\begin{array}{l}\text { Raddusa Staz. } \\
\text { Raffadali }\end{array}$ & $\begin{array}{l}\text { 269.IV.SW } \\
\text { 267.III.SW }\end{array}$ & $\begin{array}{l}\text { Stazione ff.ss. } \\
\text { Via Fontanelle 54, vicino al torrione } \\
\text { (p.to trigon.) }\end{array}$ & $\begin{array}{l}\text { p.d.f. } \\
\text { p.to trigon. }\end{array}$ & $\begin{array}{l}171 \\
201\end{array}$ \\
\hline Ragattisi & 257.IV.SW & Stazione ff.ss. & p.d.f. & 80 \\
\hline Ragusa & 276.I.NW & Stazione ff.ss. & p.d.f. & 277 \\
\hline Ramacca & 269.III.SE & $\begin{array}{l}\text { Davanti alla facciata della Chiesa della } \\
\text { Concezione, presso il marciapiede }\end{array}$ & p.to trigon. & 207 \\
\hline Randazzo & 262.IV.SW & Stazione ff.ss. & p.d.f. & 84 \\
\hline Regalbuto & 269.IV.NE & $\begin{array}{l}\text { Sulla S.S. } 121,2 \mathrm{~m} \text { ad Est ponticello } \\
\text { n. } 172 \text { al } \mathrm{km} 60,654\end{array}$ & p.to quotato & 142 \\
\hline Resuttano & 268.IV.NW & $\begin{array}{l}\text { Curva al km 5,2 della strada che con- } \\
\text { duce al paese, presso Portella del } \\
\text { Morto }\end{array}$ & p.to quotato & 136 \\
\hline Ribera & 266.II.NW & Stazione ff.ss. (sul marciapiede) & p.d.f. & 184 \\
\hline Riesi & 272.IV.NE & $\begin{array}{l}\text { Bivio, a km } 1,5 \text { ad Est del paese, per } \\
\text { Case Zagarella }\end{array}$ & p.to quotato & 228 \\
\hline Roccamena & 258.IV.SE & $\begin{array}{l}\text { Sul marciapiede, presso il bordo, davan- } \\
\text { ti la scalinata dell'ingresso principa- } \\
\text { le alla Chiesa di S. Salvatore }\end{array}$ & cs.v. IGM & 91 \\
\hline Roccapalumba & 259.III.NE & Stazione ff.ss. & p.d.f. & 104 \\
\hline Rosolini & 277.III.NW & Stazione ff.ss. & p.d. & 289 \\
\hline Roveto Bimmisca & 277.III.NE & Stazione ff.ss. & p.d.f. & 287 \\
\hline Salemi & 25i.II.NW & Piazza Cappuccini, lato Est & p.to quotato & 92 \\
\hline Salemi Staz. & 257.II.NE & Stazione ft.ss. & p.d.f. & 97 \\
\hline Sambuca & 266.IV.NE & $\begin{array}{l}\text { Davanti al cs.v. sulla facciata della casa } \\
\text { Maggio, sulla strada prov., fra le por- } \\
\text { te n. } 34 \text { e n. } 36\end{array}$ & cs.v. IGM & 141 \\
\hline Sampieri & 276.II.SW & Stazione ff.ss. & p.d.f. & 298 \\
\hline S. Biagio Platani & 267.IV.SW & $\begin{array}{l}\text { Di fronte all'ingresso della Chiesa del } \\
\text { Carmine }\end{array}$ & p.to trigon. & 179 \\
\hline S. Carlo & 266.I.NW & Stazione ff.ss. & p.d.f. & 147 \\
\hline S. Cataldo & 268.III.NW & Stazione ff.ss. & p.d.f. & 190 \\
\hline S. Caterina Vill. & 268.IV.NW & $\begin{array}{l}\text { Sulla strada, al centro della facciata del } \\
\text { nuovo edificio adibito a colonia. di } \\
\text { fianco al convento dei Cappuccini }\end{array}$ & p.to trigon. & 157 \\
\hline S. Cipirello & 258.IV.NE & $\begin{array}{l}\text { All'inizio del sentiero a } S \text { del ponte al } \\
\text { km 8,900 sulla strada Partinico-Cor- } \\
\text { leone }\end{array}$ & p.to quotato & 60 \\
\hline S. Croce Camerina & & $\begin{array}{l}\text { Davanti alla porta centrale Chiesa Ma- } \\
\text { dre, a } 5 \mathrm{~m} \text { dall'ingresso }\end{array}$ & p.to trigon. & 286 \\
\hline S. Elia & 257.III.SE & Davanti al portone a volto d'ingresso & p.to trigon. & 130 \\
\hline S. Giacomo & 266.IV.NE & $\begin{array}{l}\text { In mezzo allo spiazzo sul lato } W \text { sta- } \\
\text { zione ff.ss. }\end{array}$ & p.d.f. & 137 \\
\hline
\end{tabular}


... segue Tab. IlI

\begin{tabular}{|c|c|c|c|c|}
\hline Stazione & $F / q / t$ & Descrizione & $\begin{array}{l}\text { Riferimento } \\
\text { altimetrico }\end{array}$ & N. \\
\hline S. Giorgio & 252.I.SE & $\begin{array}{l}\text { Davanti alla facciata NW della prima } \\
\text { casa a destra appena passato il sotto- } \\
\text { passaggio della ferrovia }\end{array}$ & p.d.f. & 10 \\
\hline S. Lorenzo & 277.III.NE & Stazione ff.ss. & p.d.f. & 293 \\
\hline S. Margherita B. & 258.III.SW & $\begin{array}{l}\text { Allo spigolo destro della facciata della } \\
\text { Chiesa, a } 5 \mathrm{~m} \text { dal cs.v. }\end{array}$ & cs.v. IGM & 125 \\
\hline S. Maria Licodia & 269.I.NE & Stazione ff.ss. & p.d.f. & 149 \\
\hline S. Martino & 270.III.NW & $\begin{array}{l}\text { Ex fermata al km } 226+966 \text {, davanti } \\
\text { al cs. }\end{array}$ & cs.v. IGM & 192 \\
\hline S. Mauro Castelv. & 260.IV.NE & $\begin{array}{l}\text { Sulla strada che dalla staz. Pollina por- } \\
\text { ta al paese, davanti alla cantoniera } \\
\text { al } \mathrm{km} 75\end{array}$ & q.to quotato & 64 \\
\hline S. Michele di G. & 272.I.NE & $\begin{array}{l}\mathrm{Di} \text { fronte alla scalinata di accesso al } \\
\text { Cimitero lato } \mathrm{N} \text { della strada }\end{array}$ & cs.o. IGM & 227 \\
\hline S. Nicolò & 250.III.SE & $\begin{array}{l}\text { AI centro della facciata della Chiesa } \\
\text { parrocch. }\end{array}$ & cs.v. IGM & 48 \\
\hline S. Oliva & 271.II.NE & Stazione fi.ss. & p.d.f. & 248 \\
\hline S. Paolo & 277.1 & Stazione ff.ss. & p.d.f. & 285 \\
\hline S. Pietro & 273.III.NW & Casa di cura: al centro della facciata & p.to quotato & 257 \\
\hline S. Pietro Patti & 253.III.SW & $\begin{array}{l}\text { Case popolari sulla strada per S. Maria, } \\
\text { al centro della facciata del gruppo di }\end{array}$ & p.to quotato & 38 \\
\hline S. Stefano di C. & 251.II.SE & $\begin{array}{l}\text { tre case a Nord d } \\
\text { Stazione ff.ss. }\end{array}$ & p.d. & 46 \\
\hline $\begin{array}{l}\text { S. Stefano Quisq. } \\
\text { S. Teresa di Riva }\end{array}$ & 267.IV.NW & Stazione ff.ss. & p.d.f. & 146 \\
\hline S. Teresa di Kiva & 262.I.NE & $\begin{array}{l}\text { Sulla S.S. n. 114, di fronte all'ingresso } \\
\text { di casa Salvadore (n. } 306)\end{array}$ & cs.v. IGM & 66 \\
\hline $\begin{array}{l}\text { S. Teresa Long. } \\
\text { S. Venerina }\end{array}$ & $\begin{array}{l}\text { 274.II.SW } \\
\text { 262.III.SE }\end{array}$ & Stazione ff.ss. & $\begin{array}{l}\text { p.d.f. } \\
\text { p.to trigon. }\end{array}$ & $\begin{array}{l}269 \\
127\end{array}$ \\
\hline S. Vito Lo Capu & 248.I.SW & $\begin{array}{l}\text { Mad. Carmelo } \\
\text { Sulla piazza vicino allo spigolo SW del } \\
\text { Santuario }\end{array}$ & p.to quotato & 9 \\
\hline $\begin{array}{l}\text { Sciacca } \\
\text { Sciara }\end{array}$ & $\begin{array}{l}\text { 266.IV.SW } \\
\text { 259.I.SW }\end{array}$ & $\begin{array}{l}\text { Stazione ff.ss. } \\
\text { Sulla soglia del cancello d'ingresso al- } \\
\text { la stazione ff.ss. }\end{array}$ & $\begin{array}{l}\text { p.d.f. } \\
\text { p.d.f. }\end{array}$ & $\begin{array}{r}181 \\
75\end{array}$ \\
\hline Scic & .NE & Stazione ff.ss. & p.d.f & 290 \\
\hline Scop & 21 & $\begin{array}{l}\text { Sulla strada, presso lo spigolo SW del- } \\
\text { la Chiesa }\end{array}$ & p.to quotato & 31 \\
\hline Scordia & 273.I.NE & Stazione ff.ss. & p.d. & 223 \\
\hline Sel & & izione ff.ss. & p.d & 158 \\
\hline Sella del Contr. & & ettometro IV & & 89 \\
\hline Serradifalco & 267. & Stazione ff.ss. & & 197 \\
\hline Sferr & 269.I.SW & Stazione ff.ss. & cs.v. IGM & 183 \\
\hline & Ebis & Stazione ff.ss. & & 214 \\
\hline Sirac & 274.I & $\begin{array}{l}\text { Davanti all'uscita viaggiatori stazione } \\
\text { centrale ff.ss. }\end{array}$ & p.d.f. & 262 \\
\hline Sommatino & 272.IV.NW & $\begin{array}{l}\text { Piazza Meli: davanti all'ingresso torre } \\
\text { dell'orologio }\end{array}$ & p.to trigon. & 213 \\
\hline $\begin{array}{l}\text { Sortino } \\
\text { Spagnuola }\end{array}$ & $\begin{array}{l}\text { 274.III.NW } \\
257 \text { IV SW }\end{array}$ & Stazione ff.ss. lato binari & p.d & 250 \\
\hline $\begin{array}{l}\text { Spagnu } \\
\text { Targia }\end{array}$ & $\begin{array}{l}\text { 257.IV.SW } \\
\text { 274.II.NW }\end{array}$ & $\begin{array}{l}\text { Stazione ff.ss. } \\
\text { Stazione ff.ss. }\end{array}$ & $\begin{array}{l}\text { es.v. IGM } \\
\text { p.d.f. }\end{array}$ & $\begin{array}{r}90 \\
255\end{array}$ \\
\hline
\end{tabular}


.. segue Tab. III

\begin{tabular}{|c|c|c|c|c|}
\hline Stazione & $\mathrm{F} / \boldsymbol{q} / \mathrm{t}$ & Descrizione & $\begin{array}{l}\text { Riferimento } \\
\text { altimetrico }\end{array}$ & N. \\
\hline Termini Imerese & 259.IV.SW & $\begin{array}{l}\text { Di fronte allingresso esterno dell'Uff- } \\
\text { cio daziario, accanto al pilastro sett. } \\
\text { della Porta a Palermo }\end{array}$ & cs.o. IMG & 54 \\
\hline Terremorte & 262.III.NE & Stazione ff.ss. & p.d.f. & 93 \\
\hline Terrenove & 257.III.SW & $\begin{array}{l}\text { Lato } N \text { passaggio a livello vicino alla } \\
\text { stazione ff.ss., di fronte al cancello ca- } \\
\text { sa n. } 342\end{array}$ & p.d.f. & 113 \\
\hline Torre de Leva & 276.I.SE & $\begin{array}{l}\text { A } 5 \text { m davanti al portone d'ingresso del- } \\
\text { la casa principale (n. 89) del grup- } \\
\text { po, ad E di Modica }\end{array}$ & p.to trigon. & 283 \\
\hline $\begin{array}{l}\text { Torrenova } \\
\text { Trapani }\end{array}$ & 252.III.NE & Stazione ff.ss. & p.d.f. & 23 \\
\hline $\begin{array}{l}\text { Trapani } \\
\text { Trecastagni }\end{array}$ & $\begin{array}{l}\text { 248.III.SW } \\
\text { 271.IV.NE }\end{array}$ & $\begin{array}{l}\text { Stazione ff.ss. } \\
\text { Sul ciglio della strada per Zafferena, } \\
\text { in frazione S. Alfio, presso il bivio, } \\
\text { di fronte alla più alta del gruppo di } \\
\text { tre case ad Est (n. 26) }\end{array}$ & $\begin{array}{l}\text { es.v. IGM } \\
\text { p.to quotato }\end{array}$ & $\begin{array}{r}47 \\
148\end{array}$ \\
\hline Tremestieri & 254.III.NW & $\begin{array}{l}\text { Davanti al marciapiede di casa Artuso } \\
\text { (n. 211) sulla piazza, di fronte al cs. } \\
\text { v. IGM }\end{array}$ & cs.v. IGM & 18 \\
\hline Troina & 261.III.NW & $\begin{array}{l}\text { S.S. 120: al centro del bivio della stra- } \\
\text { da nuova per la diga di Ancipa, che } \\
\text { si distacea al km } 133\end{array}$ & p.to quotato & 99 \\
\hline Ummari & 257.I.NW & Stazione ff.ss. & p.d.f. & 70 \\
\hline Valledolmo & 259.II.SW & Stazione ff.ss. & p.d.f. & 120 \\
\hline Vallelunga & 259.II.SE & Stazione ff.ss. & p.d.f. & 126 \\
\hline Valsavoia & 270.III.SW & Stazione ff.ss. & es.v. IGM & 212 \\
\hline Verdura & 266.III.NE & Stazione ff.ss. & p.d.f. & 186 \\
\hline Villa Acampora & 270.III.NW & $\begin{array}{l}\text { Davanti al cancello, sulla strada Cata- } \\
\text { nia - Valguarnera }\end{array}$ & cs.v. IGM & 191 \\
\hline Villadoro & 260.II.SW & $\begin{array}{l}\text { Sulla strada da Nicosia, circa } 500 \mathrm{~m} \text { pri- } \\
\text { ma del paese nel punto dove si di- } \\
\text { stacca il sentiero per la Portella Creta }\end{array}$ & p.to quotato & 121 \\
\hline Villafranca & 253.1.SE & Stazione ff.ss. & p.d.f. & 3 \\
\hline Villafrati & 259.IV.NE & $\begin{array}{l}\text { Sul piazzale fra il magazzino e lo scalo } \\
\text { merci ff.ss, a } 2 \mathrm{~m} \text { dal magazzino }\end{array}$ & p.d.f. & 74 \\
\hline Villagrazia & 249.II.SE & $\begin{array}{l}\text { Presso il marciapiede di fronte alla } \\
\text { spalletta destra della scaletta d'ingres. } \\
\text { so alle scuole elem. }\end{array}$ & cs.o. IGM & 30 \\
\hline Villalba & 259.II.SE & Stazione ff.ss. & p.d. & 132 \\
\hline Villarosa & 268.IV.SW & $\begin{array}{l}\text { Al centro facciata (lato Nord) scalo } \\
\text { merci ff.ss. }\end{array}$ & p.d.f. & 163 \\
\hline Villa Scucce & 276.II.NW & $\begin{array}{l}\text { Davanti al cancello (n. 6) della villa si- } \\
\text { ta sulla strada che conduce a Cava } \\
\text { d'Ispina, dipartendosi dalla Modica- } \\
\text { Ispica al } \mathrm{km} 344+\text { VI }\end{array}$ & p.to trigon. & 288 \\
\hline $\begin{array}{l}\text { Villasmundo } \\
\text { Vittoria }\end{array}$ & $\begin{array}{l}\text { 274.IV.SE } \\
\text { 276.IV.NW }\end{array}$ & Davanti alla porta d'ingresso alla Chiesa & $\begin{array}{l}\text { p.to quotato } \\
\text { p.d.f. }\end{array}$ & $\begin{array}{l}233 \\
275\end{array}$ \\
\hline Vizzini & 273.I.SW & $\begin{array}{l}\text { Stazione ff.ss. } \\
\text { Stazione ff.ss. }\end{array}$ & p.d.f. & 244 \\
\hline Xirbi & 268.IV.SE & Stazione ff.ss. Caltanissetta-Xirbi & p.d.f. & 174 \\
\hline
\end{tabular}


gione interna della Sicilia, dove spesso la rete stradale è inesistente o in cattive condizioni.

Il rilievo di dettaglio è stato eseguito per irradiamento, sempre in andata e ritorno, dalle varie stazioni di riferimento, che sono state occupate seguendo il periplo dell'Isola in senso antiorario, con partenza e chiusura a Palermo.

La precisione delle misure di dettaglio è dell'ordine di t- $0.2 \mathrm{mgal}$. Essa viene anche confermata dalla ripetizione di alcune di esse in epoche differenti, sia partendo dalla stessa stazione di riferimento clue partendo da stazioni di riferimento diverse (v. Tabella IV).

Come si vede lo scarto massimo osservato è + 0.33 mgal (Lercara), e la precisione sopra indicata viene così confermata, e risulta più che sufficiente qualora si consideri l'incertezza derivante ai valori ridotti per l'imprecisione delle quote e delle correzioni ( $v$. $\$ 5$ ).

I valori finali della gravità osservata sono riportati nella Tabella $\mathrm{V}$, unitamente alla quota delle stazioni ed alle coordinate geografiche $p$ e $\lambda$, dedotte dalle carte 1:25.000 e corrette per gli aggiustamenti (successivi alla stampa delle tavolette) della rete geodetica del $1^{\circ}$ ordine, secondo $\mathrm{i}$ dali gentilmente comunicati dal prof. Boaga.

5. Riduzione dei valori osservati. Anomalie secondo Bouguer generalizzate. -- Abbiamo già ampiamente discusso in altra sede $\left({ }^{4}\right) \mathrm{i}$ criteri di riduzione dei valori osservati della gravità, le modalità di calcolo e le cause di errore, proponendo anche nuovi metodi e tabelle. Ci limiliamo quindi a seguire gli stessi criteri per il calcolo delle riduzioni in aria libera $\delta g$ per la calotta sferica e per la correzione topografica $g^{\prime} \cdot g$, precisando che:

a) il calcolo per la calotta sferica è stato esteso fino alla zona $O$. di Hayford ( $\mathrm{km} 166.7$ dalla stazione);

b) la distribuzione delle densità adottata è quella indicata dalla fig. 3 ;

c) la correzione topografica è stata arrestata alla zona $L$ (kna 28.8), dato che essa diventa praticamente trascurabile per le zone successive a questa ( $\left.{ }^{4}\right)$;

d) entro questi limiti, per i compartimenti in tutto o in parte occupati dal mare, si è tenuto anche conto del difetto di densità che ad esso compete (correzione di Bouguer generalizzata).

I risultati dei calcoli sono riportati nella seconda metà della Tabella $\mathrm{V}$, e la rappresentazione grafica delle isoanomale secondo Bouguer nella fig. 4 . 


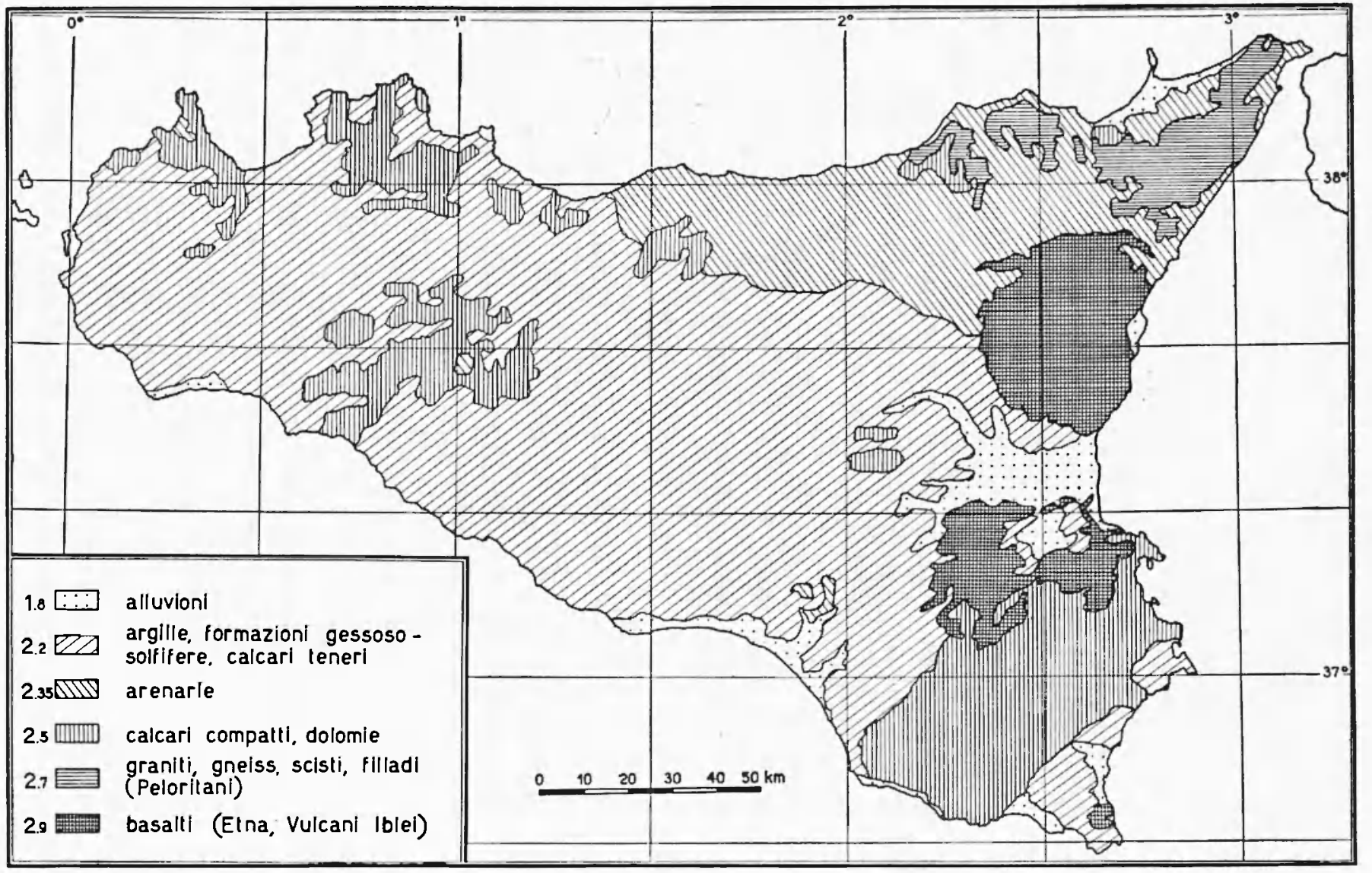

Fig. 3 - Differenziazione schematica della densità, per il calcolo della correzione per la calotta e topografia 
I calcoli sono stati arrestati alla riduzione di Bouguer, in quanto è questa che rappresenta il maggior interesse per la prospezione geofisica, e dato che per accordi intercorsi con la Commissione Geodetica Italiana, il calcolo della riduzione isostatica è stato da questa assunto per tutta l'Italia.

6. Discussione dei risultati. - L'esame della fig. 4 mostra anzitutto chiaramente che il contributo degli errori di osservazione e di riduzione è praticamente trascurabile: ciò è indicato dalla forma in generale regolare delle isoanomale, in particolare di quelle che si riferiscono all'anomalia negativa centrale.

Inoltre, il quadro delle anomalie stesse è talmente marcato, che eventuali variazioni derivanti da piccole differenze nelle densità assunte per il calcolo della correzione per la calotta (fig. 3 ) non possono modificare sensibilmente l'andamento generale delle anomalie stesse: queste hanno quindi un significato fisico ben definito, che non viene sostanzialmente modificato neppure dalla riduzione isostatica $\left({ }^{5}\right)$.

Resta ora da precisare qual'è questo significato fisico. Quantunque ciò non rientri fra $i$ fini della presente relazione, vogliamo farne brevemente cenno, per l'interesse che il problema riveste.

Né una trattazione più ampia avrebbe qui significato, per essere il problema già ampiamente stato studiato circa 20 anni fa dal prof. Fabiani, ed ora nuovamente e minuziosamente elaborato dal dott. Beneo $\left({ }^{6}\right)$ e dalle Società di prospezione che numerose stanno operando in Sicilia. Delle pubblicazioni in argomento del prof. Fabiani e dei suoi collaboratori citiamo quella riassuntiva $\left({ }^{7}\right)$, che riporta in bibliografia le altre.

Il quadro gravimetrico della Sicilia è caratterizzato (v. fig. 4) dall'estesa anomalia negativa centrale sopra menzionata, estendentesi in mare verso Sud e circondata per il resto da anomalie positive, notevoli soprattutto verso SE. Poiché, come si è detto, tale quadro non viene sostanzialmente mutato né dai diversi valori assunti per le densità nelle riduzioni dei valori osservati della gravità, né dai diversi tipi di riduzioni applicate, anche isostatiche, esso non può essere attribuito solo alle stratificazioni più esterne della crosta, ma rispecchia probabilmente caratteristiche degli strati più profondi di questa.

E così probabile che la grande anomalia negativa corrisponda ad un'ampia fossa di origine tettonica gradualmente aftondantesi, nella quale si sono venuti accumulando in un modo o nell'altro sedimenti 
Tabella IV

Scarti osservati nella ripetizione dei punti di dettaglio.

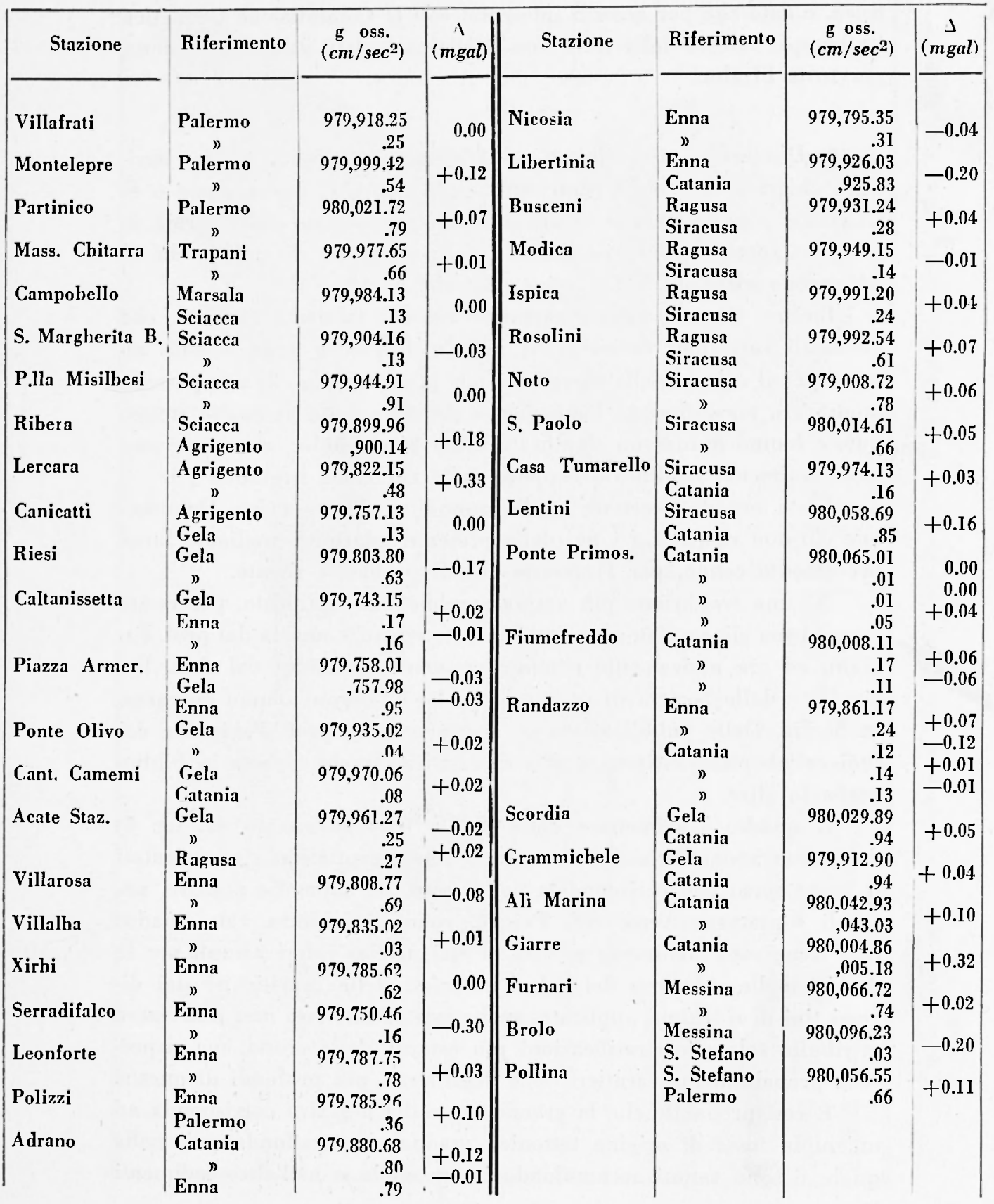




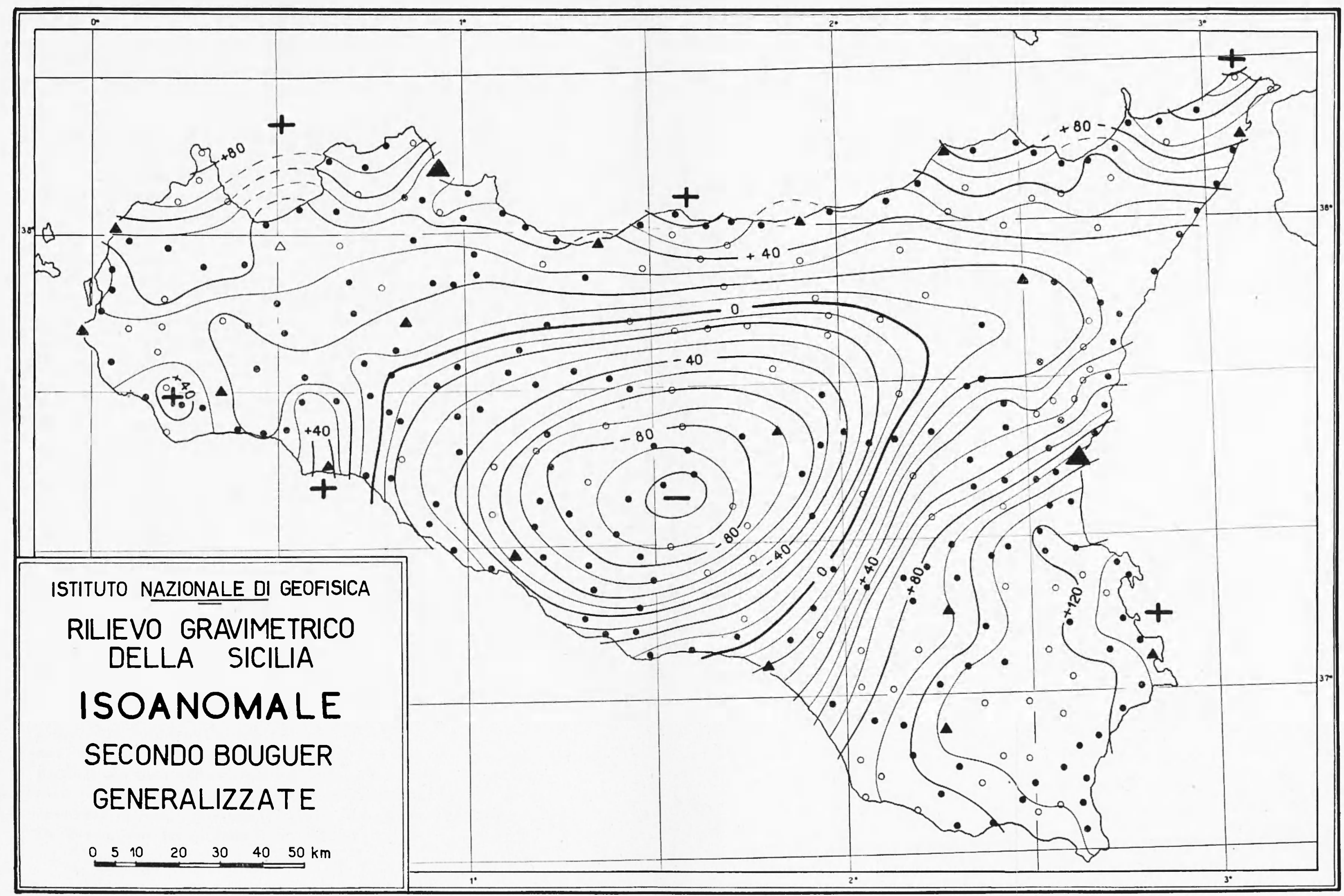


a bassa densità. Tuttavia la presenza di grandi blocchi calcarei, quali i M. Sicani, affioranti proprio nell'area depressa, dà sufficiente ragione all'ipotesi dell'accumulo per traslazione, secondo l'ipotesi di Beneo.

Secondo questo A. infatti ("). in Sicilia l'autoctono supposto in prevalenza calcareo affiora esclusivamente nella parte orientale dell'Isola (altipiano di Ragusa), mentre il resto è ricoperto da una estesa massa plastica alloctona prevalentemente argillosa, costituente l'unità tettonica denominata "argille scagliose" (A.S.) e contenente esotici, più rigidi, di ogni dimensione e delle più diverse età. Autoctoni sono ancora almeno in parte i Monti Peloritani (metamorfici), l'Etna ed i Vulcani Iblei.

L'ipotesi da noi sopra avanzata $\dot{e}$ in accordo e giustifica queste vedute: la grande massa dei terreni plastici della coltre di A.S. occuperebbe col massimo spessore l'area di depressione, mentre sareble più esigua o nulla in corrispondenza degli affioramenti di terreni autoctoni. Il meccanismo è reso pienamente intuitivo se si tiene presente che $\left({ }^{6}\right)$ " le A.S. della Sicilia e tutte le A.S. del mondo, recenti o antichissime, si comportano come una massa fluida che cola su un substrato rigido per effetto delle infinite componenti tangenziali della gravità. Questa massa plastica, instabile, si muove dai bacini di origine, di qualsiasi età, triassica o cretacea o terziaria, (se ci si riferisce all'orogenesi alpina), avanzando a più riprese nel tempo e nello spazio, sollecitata da una vera e propria onda orogenetica. In realtà si verifica che in tempi enormi ha percorso spazi forse relativamente limitati ma di ampiezza varial,ilissima ).

L'effetto massimo di innalzamento del substrato denso si ha nel SE dell'Isola, dove le masse effusive note e le intrusive ancora per la massima parte ignote degli Iblei ne costituiscono l'effetto più cospicuo. Secondo Beneo ( ${ }^{\circ}$ ), l'altopiano calcareo di Ragusa, autoctono, è stato aggirato come se fosse un bastione o uno scoglio dalle A.S. nella loro traslazione (sottomarina), con verso approssimativo $\mathrm{N}-\mathrm{S}$.

Si nota poi in generale (v. fig. 4) che l'andamento delle isoanomale positive è molto più movimentato di quello delle isonomale negative della zona centrale dell'Isola: ciò perché queste derivano da una causa più profonda. Così l'anomalia positiva regionale che corre parallela alla costa settentrionale dell'Isola presenta numerose diminuzioni (anomalie negative locali). Queste sono probabilmente in corrispondenza di fosse trasversali che, come ci comunica gentilmente il Dr. Beneo, sono invase dalla coltre alloctona. 
TABella V - Coordinate delle stazioni e valori della gravità osservata; valori normali, corre. zioni ed anomalie secondo Faye e secondo Bouguer generalizzate.

\begin{tabular}{|c|c|c|c|c|c|c|c|c|c|c|}
\hline \multirow{2}{*}{ N. Stazione } & \multirow{2}{*}{ ० } & \multirow{2}{*}{$\underset{(\underset{0}{\text { (Greennw/ch) }})}{\lambda}$} & \multirow{2}{*}{$\underset{(m)}{\mathbf{h}}$} & \multirow{2}{*}{$\stackrel{g}{g}$} & \multirow{2}{*}{$\begin{array}{c}\gamma_{\mathrm{n}}^{\prime} \\
(\mathrm{gal})\end{array}$} & \multicolumn{3}{|c|}{ Correzioni (mgal) } & \multicolumn{2}{|c|}{ Anomalie ( $m g a l$ ) } \\
\hline & & & & & & ig & calotta & $g^{\prime} \cdot g$ & $\mathbf{g}_{0}=\gamma_{0}{ }^{\circ}$ & $g^{\prime \prime} 0-\gamma 0^{\circ}$ \\
\hline 1. Aquarone & 38.17 .33 & 15.32 .10 & 94 & $980,126.0$ & $980,029.8$ & +29.0 & -7.2 & +1.4 & +125.3 & +119.5 \\
\hline 2. Capo Peloro & 16.02 & 39.08 & 3.0 &, 116.2 &, 027.5 & 0.9 & 0.3 & 1.1 & 89.6 & 90.4 \\
\hline 3. Villafranca & 13.59 & 25.50 & 27.6 & , 122.7 & 024.5 & 8.5 & 2.7 & 2.0 & 106.7 & 106.0 \\
\hline 4. Milazzo & 12.41 & 14.14 & 4.1 & 110.0 &, 022.6 & 1.3 & 0.3 & 1.6 & 88.7 & 89.9 \\
\hline 5. Monforte Mar. & 25 & 20.33 & 12.5 & , 109.2 & ,022.2 & 3.9 & 1.0 & 1.6 & 90.8 & 91.4 \\
\hline 6. Partanna Mond. & 11.44 & 13.18 .45 & 11 &, 094.2 &, 021.2 & 3.4 & 1.0 & 1.3 & 76.4 & 76.7 \\
\hline 7. Isola d. Femm. & 40 & 15.02 & 17.2 & ,093.8 & ,021.1 & 5.3 & 1.5 & 1.9 & 78.0 & 78.4 \\
\hline 8. Messina & 16 & 15.33.35 & 3.2 & , 104.9 & $, 020,5$ & 1.0 & 0.2 & 1.4 & 85.3 & 86.5 \\
\hline 9. S. Vito Lo Capo & 10.31 & 12.44 .05 & 6 & , 103.5 &, 019.5 & 1.9 & 0.5 & 0.7 & 85.9 & 86.2 \\
\hline 10. S. Giorgio & 26 & 14.56 .43 & 2.5 & , 100.1 & ,019.3 & 0.8 & 0.2 & 4.3 & 81.5 & 85.6 \\
\hline 11. Brolo & 38.09 .29 & 14.50 .04 & 7.2 & $980,096.1$ & $980,018.0$ & +2.2 & 0.6 & +3.4 & +80.3 & -83.2 \\
\hline 12. Capo Orlando & 27 & 44.47 & 10.7 & 092.2 &, 017.9 & 3.3 & 1.1 & 2.6 & 77.6 & 79.1 \\
\hline 13. Monforte & 23 & 15.22 .55 & 260 &, 047.8 &, 017.8 & 80.2 & 19.8 & 2.6 & 110.2 & 93.0 \\
\hline 14. Cinisi & 17 & 12.55 .51 & 47.4 &, 074.7 & $, 017,7$ & 14.6 & 4.6 & 1.3 & 71.7 & 68.4 \\
\hline 15. Barcellona & 08.54 & 15.12 .50 & 45.1 & ,071.4 & 017.1 & 13.9 & 4.0 & 0.9 & 68.2 & 65.1 \\
\hline 16. Carini & 49 & 13.11 .15 & 48.3 &, 076.7 &, 017.0 & 14.9 & 4.3 & 1.8 & 74.6 & 72.1 \\
\hline 17. Patti & 45 & 14.58 .36 & 12.2 & 088.6 &, 016.9 & 3.8 & 1.1 & 3.0 & 75 & 77.4 \\
\hline 18. Treinestieri & 15 & 15.31 .33 & 14.3 &, 086.5 &, 016.2 & 4.4 & 1.3 & 3.2 & 8 & 76.6 \\
\hline 19. Furnari & 07.15 & 07.54 & 23.1 & 066.7 &, 014.7 & 7.1 & 1.8 & 1.8 & 59.1 & 59.2 \\
\hline 20. Case Fontanotti & 08 & 12.44 .05 & 27 & 079.5 &, 014.5 & 8.3 & 2.5 & 2.6 & 73.3 & 73.4 \\
\hline 21. Falcone & 38.07 .06 & 15.05 .00 & 1.7 & $0,074,1$ & $980,014.5$ & +0.5 & -0.1 & +2.0 & +60.1 & +62.0 \\
\hline 22. Palermo V. G. & 06.47 & 13.22 .39 & 2.7 &, 057.4 &, 014.0 & 0.8 & 0.3 & 1.0 & 44.2 & 44.8 \\
\hline 23. Torrenova & 05.35 & 14.40 .39 & 6.5 & ,062.1 &, 012.3 & 2.0 & 0.7 & 2.8 & 51.8 & 53.9 \\
\hline 24. Montelepre & 05.19 & 13.10 .23 & 343 & $979,999.5$ &, 011.9 & 105.8 & 34.8 & 2.9 & 93.4 & 61.4 \\
\hline 25. Ficarazzi & 18 & 28.16 & 23.3 & $980,059.6$ & 011.9 & 7.2 & 1.8 & 0.5 & 54 & 53.7 \\
\hline 26. Cast & 17 & 15.12 .04 & 328 &, 003.4 & 011.9 & 101.2 & 34.7 & 1.7 & 92.7 & 59.7 \\
\hline Imberto & 01 & 14.48 .25 & 631 & $979,938.6$ &, 011.5 & 194.7 & 64.1 & 5.1 & 121.9 & 62.9 \\
\hline 28. Mon & 04.52 & 13.17 .31 & 301 &, 997.9 &, 011.2 & 92.9 & 30.6 & 2.7 & 79.5 & 51.6 \\
\hline 29. Cust & 38 & 12.40 .36 & 176.8 & $980,046.0$ &, 010.9 & 54.6 & 13.5 & 0.7 & 89.7 & 76.8 \\
\hline 30. Villagrazia & 35 & 13.19.01 & 130.0 &, 028.3 &, 010.8 & 40.1 & 12.7 & 2.4 & 57.6 & 47.4 \\
\hline 31. Sco & 38.04 .10 & 12.50 .09 & 106 & $980,045.3$ & $980,010.2$ & +32.7 & -10.3 & 2.3 & +67.8 & +59.8 \\
\hline 32. Gi & 03.46 & 15.28.55 & 6.2 &, 064.3 &, 009.6 & 1.9 & 0.7 & 8.7 & 56.6 & 64.7 \\
\hline 33. Bas & 38 & 03.44 & 528 & $979,953.8$ &, 009.4 & 162.9 & 49.2 & 2.2 & 107.3 & 60.4 \\
\hline 34. Par & 27 & 13.06.35 & 141.0 & $980,021.8$ &, 009.2 & 43.5 & 14.0 & 0.7 & 56.1 & 42.8 \\
\hline 35. Acquedolci & 27 & 14.35.21 & 11.3 & .7 & 009.2 & 3.5 & 1.2 & 3.9 & 42.1 & 44.8 \\
\hline 36. Balestrate & 10 & 13.00 .29 & 22.3 & 037.5 &, 0 & 6.9 & 2.3 & 0.7 & 35.6 & 34.1 \\
\hline 37. Belmonte & 02.52 & 23.15 & 346.7 & $979,978.1$ &, 008.3 & 107.0 & 27.9 & 4.1 & 76.7 & 53.0 \\
\hline 38. S. Pietro Patti & 52 & 14.58 .37 & 538 &, 947.6 &, 008.3 & 166.0 & 56.9 & 3.5 & 105.3 & 51.8 \\
\hline 39. Altavilla & 50 & 13.33.19 & 7.6 & $980,053.3$ &, 008.3 & 2.3 & 0.8 & 1.0 & 47.4 & 47.6 \\
\hline 40. Caronia & 01 & 14.26 .42 & 19.6 & ,049.8 & ,007.1 & 6.1 & 1.9 & 3.3 & 48.8 & 50.1 \\
\hline 41. Misilmeri & 38.01 .56 & 13.27 .10 & 117.9 & $980,022.4$ & $980,006.9$ & +36.4 & -13.0 & & +51.8 & $1+40.2$ \\
\hline 42. Cefalù & 56 & 11.01 .13 & 23.0 & &, 006.9 & 7.1 & 1.8 & $\begin{array}{r}T \\
\quad 2.8\end{array}$ & $\begin{array}{r}+51.0 \\
64.2\end{array}$ & 65.3 \\
\hline 43. Longi & 32 & 45.17 & 609 & $979,915.2$ &, 006.4 & 187.9 & 49.0 & 8.2 & 96.8 & 56.0 \\
\hline
\end{tabular}


... segue TAB. V

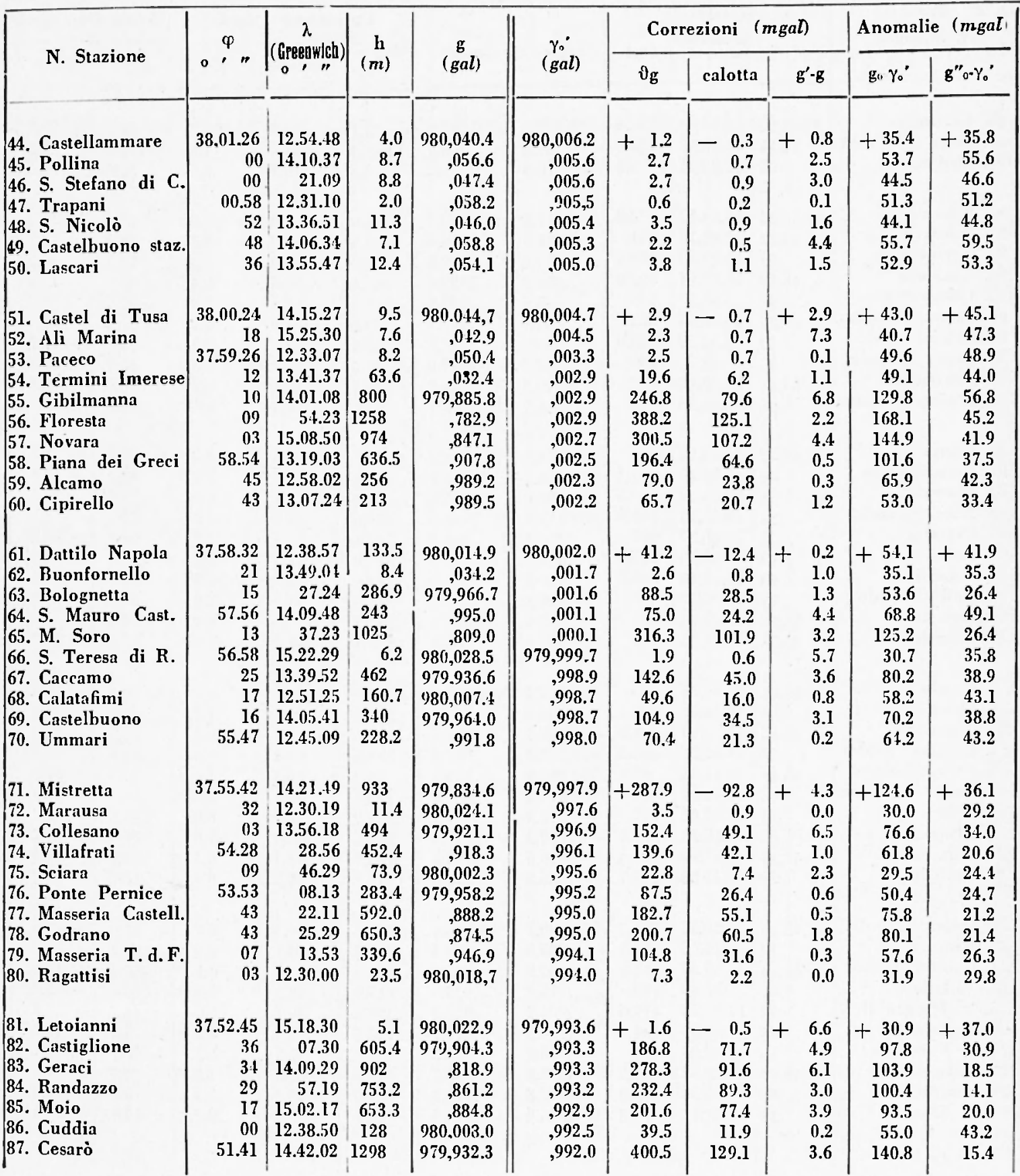


... segue TAB. V

\begin{tabular}{|c|c|c|c|c|c|c|c|c|c|c|}
\hline \multirow{2}{*}{ N. Stazione } & \multirow{2}{*}{$0, \stackrel{\varphi}{\prime}$} & \multirow{2}{*}{$\left|\begin{array}{c}{ }^{\prime} \\
\text { (Gresnwich) } \\
0\end{array}\right|$} & \multirow{2}{*}{$\underset{(m)}{\mathbf{h}}$} & \multirow{2}{*}{$\underset{(\text { gal })}{\mathrm{g}}$} & \multirow{2}{*}{$\begin{array}{c}\gamma_{0^{\circ}} \\
(\text { gal })\end{array}$} & \multicolumn{3}{|c|}{ Correzioni (mgal) } & \multicolumn{2}{|c|}{ Anomalie (mgal) } \\
\hline & & & & & & $\theta g$ & calotta & $g^{\prime} \cdot g$ & $\mathrm{~g}_{0}-\gamma_{0}^{\prime}$ & $g^{\prime \prime} 0_{0}-\gamma^{\sigma^{*}}$ \\
\hline 88. Gallitello & 37.51 .25 & 12.57 .00 & 136.4 & $9,990.8$ & $979,991.6$ & +42.1 & 12.7 & $+\quad 0.2$ & -41.3 & +28.8 \\
\hline 89. Sella d & 50.54 & 14.23 .52 & 1120 &, 759.6 & 990.9 & 345.6 & 111.4 & 1.9 & 114.3 & 4.8 \\
\hline 90. Spagnuola & 07 & $|12.28 .41|$ & 5.3 & $980,020.0$ & ,989.7 & 1.6 & 0.4 & 0.0 & 32.0 & 31.6 \\
\hline 91. Roccamena & 37.50 .06 & 13.09.17 & 472.8 & $979,911.4$ & $979,989.7$ & +145.9 & 44.0 & +1.0 & +67.6 & +24.6 \\
\hline 92. & 49.22 & 12.48 .16 & 335 &, 933.5 & ,988.6 & 103.4 & 31.9 & 0.8 & 48.3 & 17.1 \\
\hline 93. $\mathrm{T}$ & 21 & 15.09 .20 & 489.5 & ,927.0 & ,988.6 & 151.0 & 55.9 & 3 & 89.4 & 37.4 \\
\hline 94. 0 & 48.40 & 13.18 .10 & 542.6 & ,888.9 & 987.6 & 167.4 & 52.8 & 1 & 68.7 & 17.3 \\
\hline 95. C & 36 & 53.57 & 685 &, 843.6 & ,987.5 & 21 & 68.1 & 1 & 67.4 & 0.5 \\
\hline ia Salam. & 27 & 14.26 .07 & 822 &, 798.9 & ,987.3 - - - & 253.6 & 80.0 & 1 & 65.3 & -13.7 \\
\hline 97. Salemi staz. & 21 & 12.51 .59 & 223.0 &, 959.8 &, 987.1 & 68.8 & 20.8 & 0 & 41.5 & +20.9 \\
\hline Lintarra & 15 & 38.25 & 149 & .977 .7 & 987.0 & 46.0 & 13.9 & 0 & 36.7 & 23.0 \\
\hline 99. $\mathrm{T}_{\mathrm{I}}$ & 47.47 & 14.42 .48 & 980 &, 777.8 & .3 & 302.4 & 95.4 & 0 . & 93.9 & -0.8 \\
\hline 100. Masseria Spanò & 46 & 12.44 .13 & 125 & ,984.5 & ,986.3 & 38.6 & 11.6 & 0.1 & 36.8 & +25.3 \\
\hline 101. & 37.47 .42 & 14.24 .11 & 797 & 979 & $979,986.2$ & +245.9 & -77.6 & +0.8 & +54.6 & -22.3 \\
\hline freddo & & 15.23 .39 & 65.1 & $980,008.1$ & ,986.1 & \begin{tabular}{|l|}
20.1 \\
\end{tabular} & 7.4 & 3 & 42.1 & +38.6 \\
\hline 103. $\mathrm{V}$ & 34 & 12.26 .39 & 9.8 &, 008.8 & ,986.0 & 3. & 0.8 & 0 & 25.8 & 25.1 \\
\hline 104. Roc & 34 & 13.51 .43 & 349.9 & $979,911.1$ &, 986.0 & 108.0 & 32.6 & & 33.1 & 1.7 \\
\hline 105. P & 22 & 14.06 .37 & 991 &, 750.1 &, 985.7 & 30 & 92.3 & 1 & 70.2 & -20.9 \\
\hline 106. L & 14 & 49.53 & 833.0 & 1 & ,985.5 & 25 & 91.7 & 2 & 96.6 & +7.8 \\
\hline $107 . \mathrm{G}$ & 03 & 12.58 .10 & 3.19 .8 &, 729.5 &, 985.2 & 10 & 32.6 & 1. & 52.2 & 21.1 \\
\hline 108. Polizzi Genor. & 01 & 14.01 .17 & 835 &, 785.3 & 985.2 & 257.6 & 77.7 & & 57.8 & -18.0 \\
\hline 109. Nocille & 46.23 & 15.07 .44 & 866 & 837.4 &, 984.3 & 267.2 & 106.3 & 6. & 120.3 & +20.9 \\
\hline 110. Nicosia & 45.57 & 14.25 .55 & 760 & ,795.4 & ,983.6 & 234.5 & 70.8 & 0.4 & 46.2 & -24.2 \\
\hline $111 . \mathrm{C}$ & 37.45 .04 & 14 & 15 & 979,9 & $979,982.4$ & +4 & -14.5 & +0 & 39.9 & +25.5 \\
\hline 112. & & 13.16 .06 & 062.5 & .0 & ,982.3 & 'T 204.4 & 64.5 & $\begin{array}{llll}T & 0 \\
\end{array}$ & 78.0 & 14.8 \\
\hline 113. ? & 44.53 & 12.28 .53 & 8.5 & $980, \mathrm{C}$ & ,982.1 & 6 & 0.7 & 0 & 26.1 & 25.4 \\
\hline Friddi & 26 & 13 & 658.0 & 2.3 & ,981.4 & 203 & 64.1 & 0. & 43.9 & -19.8 \\
\hline e-Riposto & 43.49 & 15.11 .38 & 41.9 & 4.9 & ,980.5 & 12 & 4.4 & & 37.3 & +38.4 \\
\hline Entellina & 43 & 13.11.11 & 526.2 & 979,8 & ,980.4 & 16 & 53.4 & 2 & 59.3 & 7.9 \\
\hline 117. N & 17 & 15.07 .06 & 719 & 2.1 & & & 88.2 & 6 & 114.2 & 32.8 \\
\hline 118. $\mathrm{F}$ & 03 & 13. & 870.2 & .2 & .4 & 2 & 88.4 & 1 & 76.3 & -11.2 \\
\hline 119. P & 42.54 & 12.53 .20 & 355.6 & .6 &, 97 & 10 & 33.1 & & 50.1 & +17.4 \\
\hline 120. Valledolmo & 30 & 13.45 .26 & 499.7 & ,836.4 &, 978.6 & 154.2 & 46.5 & 0.4 & 11.9 & -34.2 \\
\hline 121. I & & 13.33 .42 & 781.7 & $979,790.1$ & $79,978.4$ & +241.2 & -76.1 & +0. & +52.9 & -22.5 \\
\hline ino & 17 & 14.36 & 684.7 & 8 & 3 & $2]$ & 69.5 & 2. & 66.7 & 0.9 \\
\hline Etnea & 41.53 & 14.59 .47 & 1882.8 & ,631.9 & 977.7 & 58 & 231.1 & 10.1 & 235.1 & +14.1 \\
\hline 124. Villadoro & 46 & 16.40 & 732 &, 775.4 & ,977.6 & 225.9 & 68.2 & 0. & 23.7 & -43.7 \\
\hline 125. S. Margh. Bel. & 29 & 13.01 .29 & 419.6 & ,904.2 &, 977.2 & 129.5 & 39.1 & 0. & 56.5 & +17.8 \\
\hline 126 & 26 & 50.50 & 439.2 & 836.8 &, 977.1 & 13 & 40.9 & 0.5 & $-\quad 4.8$ & -45.2 \\
\hline 127. S. Venerina & 15 & 15.08 .08 & 359 & ,953.1 & ,976.8 & 110.8 & 42.5 & 4.2 & +87.1 & †. 48.7 \\
\hline 128. Palazzo Adr. & 40.49 & 13.22 .57 & 681.9 & ,813.5 &, 976.2 & 210.4 & 69.3 & 1. & 47.7 & -19.8 \\
\hline onovo & 48 & 38.38 & 385.8 &, 857.8 & ,976.2 & 119.0 & 38.4 & 1. & 0.7 & 36.4 \\
\hline 130. & 20 & 12.38 .17 & 59.8 & $980,006.9$ &, 975.5 & 18.5 & 5.6 & 0.0 & 49.9 & +44.3 \\
\hline
\end{tabular}


... segue TiB. V

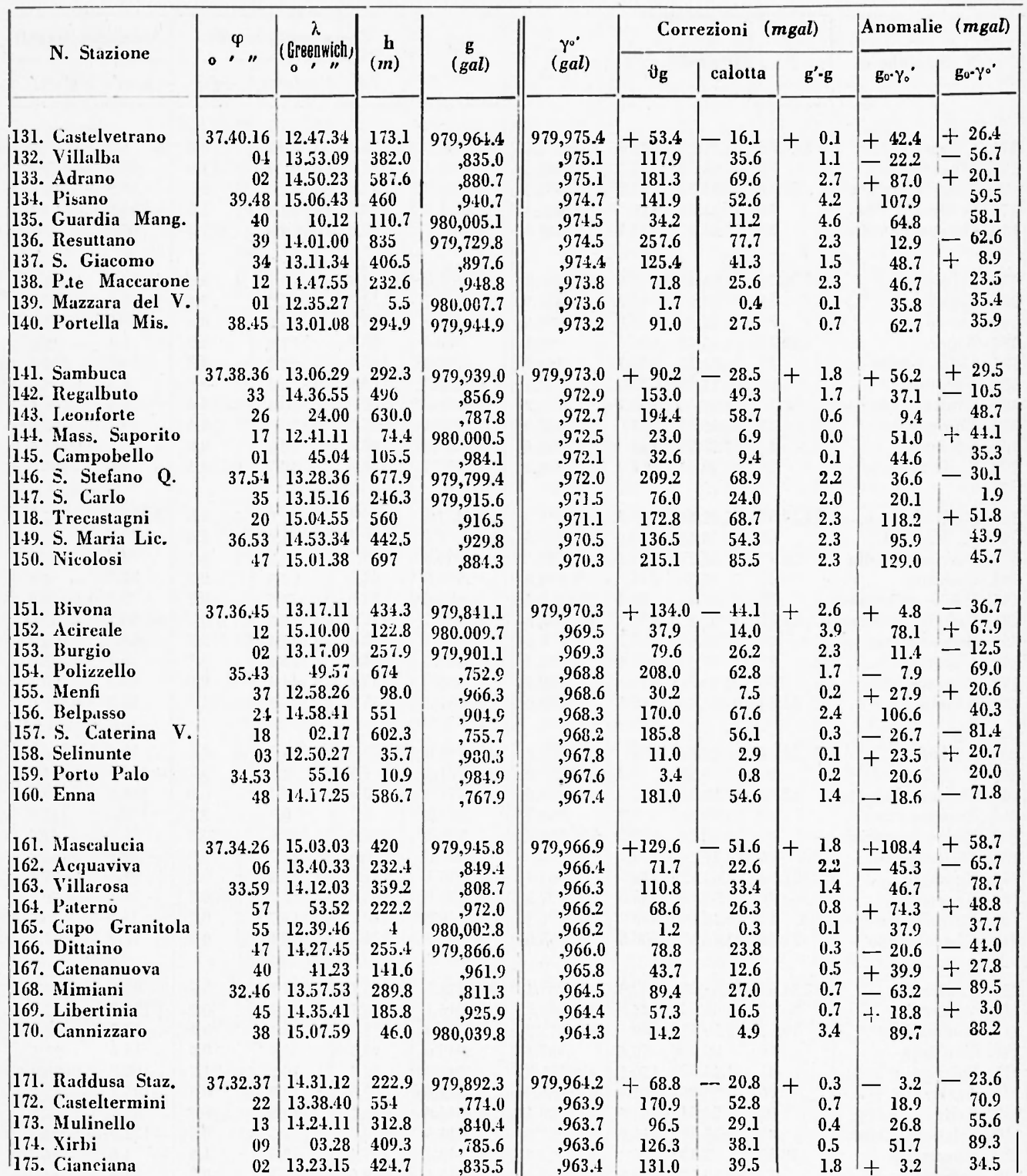


... segue $\mathrm{T}_{\mathrm{AB}} \mathrm{V}$

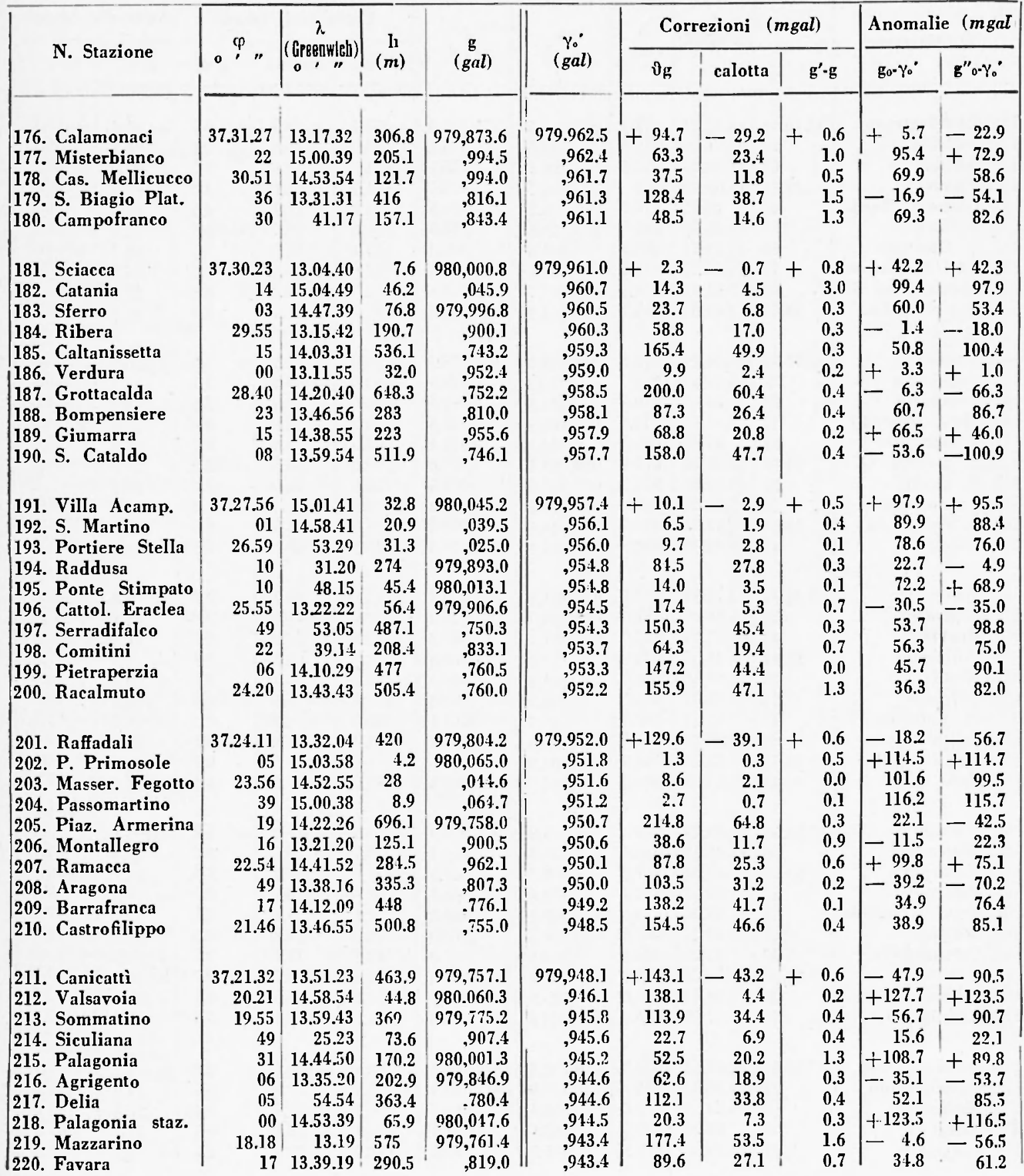


... segue Taв. V

\begin{tabular}{|c|c|c|c|c|c|c|c|c|c|c|c|}
\hline \multirow{2}{*}{ N. Stazione } & \multirow{2}{*}{$0^{P}}$, & \multirow{2}{*}{ 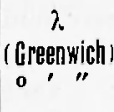 } & \multirow{2}{*}{$\underset{(\boldsymbol{m})}{\mathbf{h}}$} & \multirow{2}{*}{$\underset{(\text { gal })}{g}$} & \multirow{2}{*}{$\begin{array}{c}\gamma_{o^{\prime}} \\
\text { (gal) }\end{array}$} & \multicolumn{4}{|c|}{ Correzioni (mgal) } & \multicolumn{2}{|c|}{ Anomalie ( $m g a l$ ) } \\
\hline & & & & & & vg & calotta & & $g^{\prime} \cdot g$ & $g_{0,}, y_{0^{\circ}}$ & $g^{\prime \prime}{ }_{1}-\gamma_{\cdot . .}$ \\
\hline 221. Agnone & 37.18 .13 & 15.04 .45 & 16.9 & 980.058 .8 & $979,943.3$ & $+\quad 5.2$ & 1.9 & t- & 0.7 & +120.7 & +119.5 \\
\hline 222. Lentini & & 14.59 .53 & 26.1 &, 058.9 &, 943.0 & 8.1 & 2.4 & & 0.3 & 123.9 & 121.8 \\
\hline 223. Scordia & 17.35 & 51.11 & 110.3 &, 029.9 &, 942.4 & 34.0 & 12.6 & & 0.3 & 121.5 & 109.2 \\
\hline 224. Naro & 18 & 13.47 .43 & 419.7 & $979,778.1$ &, 942.0 & 129.5 & 39.1 & & 1.7 & -34.4 & $-\pi 1.8$ \\
\hline 225. Porto Emped. & 10 & 31.32 & 4.4 & ,905.4 &, 941.8 & 1.4 & 0.4 & & 1.7 & 35.1 & 33.7 \\
\hline 226. Fondacazzo & 16.51 & 14.40 .47 & 176.3 &, 989.3 & ,941.4 & 54.1 & 13.7 & & 1.1 & +102.3 & +81.7 \\
\hline 227. S. Micl. di G. & 48 & 26.16 & 473.7 &, 846.7 &, 941.3 & 146.2 & 44.1 & & 1.8 & 51.6 & 9.2 \\
\hline 228. Riesi & 34 & 05.50 & 326 &, 803.7 &, $9+1.0$ & 100.6 & 30.1 & & 0.1 & -36.6 & -66.9 \\
\hline 229. Brucoli & 20 & 15.11 .18 & 49.9 & $980,064.8$ &, 940.6 & 15.4 & 5.1 & & 2.0 & 139.6 & +136.6 \\
\hline 230. Campob.-Rav. & 15.52 & 13.56 .51 & 295.8 & $979,804.8$ &, 939.9 & 91.3 & 27.5 & & 0.2 & 43.8 & -71.2 \\
\hline 231. Cint & 37.15 .27 & 14.37.25 & 206 & $979,970.1$ & $979,939.4$ & 63.6 & 19.2 & t & 0.5 & $+94 . \hat{\jmath}$ & +75.6 \\
\hline 232. C & & 13.47 .31 & 342.7 &, 799.2 & 939.0 & 105.7 & 31.9 & & 0.1 & - & -65.9 \\
\hline 233. Villasmundo & 14.58 & 15.05 .36 & 191 & $980,023.4$ &, 938.7 & 58.9 & 22.6 & & 0.5 & +143.7 & +121.5 \\
\hline 234. Mineo & 41 & 14.46 .00 & 577.9 & 979.921 .4 &, 938.3 & 178.3 & 68.5 & & 0.8 & 161.4 & 93.8 \\
\hline 235. Augusta & 33 & 15.13 .09 & 4.1 & $980,073.5$ &, 938.1 & 1.3 & 0.4 & & 2.5 & 136.7 & 138.8 \\
\hline 236. Fr & 13.48 & 14.52 .51 & 277.9 & $979,997.1$ & ,937.0 & 85.7 & 32.9 & & 0.8 & .9 & 113.7 \\
\hline 237. C & 47 & 31.10 & 511.6 &, 881.0 &, 937.0 & 157.9 & 47.6 & & 0.4 & 101.9 & 54.6 \\
\hline Tumarello & 39 & 15.01 .11 & 401 &, 974.2 & ,936.8 & 123.7 & 49.2 & & 0.6 & 161.1 & 112.5 \\
\hline 239. Melilli & 12.40 & 09.17 & 29 & $980,061.3$ &, 935.4 & 9.0 & 2.3 & & 1.1 & 134.9 & 133.2 \\
\hline 2.10. Grammichele & 29 & 14.38 .31 & 500.9 & $979,912.9$ & ,935.1 & 154.6 & 50.9 & & 0.5 & 132.3 & 82.11 \\
\hline 24l. Fa & 37.12 .15 & 13.54 .51 & 130.4 & $979,857.9$ & $979,934.8$ & $\div 40.2$ & 12.1 & + & 0.9 & - & -17.9 \\
\hline 242. Passo di Piazza & 00 & 14.22 .15 & 183.9 &, 908.5 &, 934.4 & 56.7 & 17.9 & & 0.5 & +30.8 & +13.1 \\
\hline 243. Palma Montec. & 11.17 & 13.16 .35 & 179.6 & ,85.5.9 & ,933.4 & 55.4 & 16.0 & & 0.4 & -22.0 & -37.5 \\
\hline 244. Vizzini & 10.53 & $14.4+1.21$ & 577.2 &, 913.9 &, 932.8 & 178.1 & 70.8 & & 0.2 & +159.2 & +88.5 \\
\hline 245. Butera & 35 & 11.47 & 205 & 87 &, 932.4 & 63.3 & 19.1 & & 0.5 & 3.2 & -15.5 \\
\hline 2.46. Priolo & 09.26 & 15.11 .54 & 7.3 & $980,067.1$ & 0.7 & 2.3 & 0 . & & 1 & 138.7 & +139.1 \\
\hline 247. Punta Ciotta & 25 & 13.48 .59 & 122.3 & $979,877.5$ & .930 .7 & 37.8 & 9.3 & & 0.8 & -15.1 & -23.9 \\
\hline 248. S. Oliva & 22 & 54.10 & 78.6 &, 888.6 &, 930.6 & 24.3 & 7.3 & & 0.5 & 17.7 & 24.5 \\
\hline 249. Buccheri & 00 & 14.49 .51 & 717.1 & .395 .8 & 930.1 & 221.3 & 85.0 & & 1.0 & +187.0 & +103.0 \\
\hline 250. Sortino & 08.48 & 15.03 .23 & 180.5 & 980.016 .1 &, 929.8 & 55.7 & 20.6 & & 2.3 & 142.1 & $123 . \overline{7}$ \\
\hline 251. N & 37.08 .41 & 14.23 .11 & 332 & 979.885 .9 & 979 & 102.1 & 32.3 & + & 1.1 & -58.7 & +27.8 \\
\hline 252. Butera Staz. & 40 & 10.23 & 82.1 &, 905.2 & 929.6 & 25.3 & 7.3 & & 0.1 & 1.0 & -5.9 \\
\hline 253. Ponte Olivo & 03 & 18.35 & 47.8 &, 935.0 & 928.7 & 11.7 & 4.5 & & 0.2 & 21.1 & +16.8 \\
\hline 254. Falconara & 06.44 & 03.11 & 24.9 &, 916.9 &, 926.8 & 7.7 & 1.9 & & 0.3 & -2.2 & -3.8 \\
\hline 255. Targia & 32 & 15.14 .19 & 10.8 & $980,059.6$ &, 926.5 & 3.3 & 1.1 & & 2.3 & +136.4 & +137.6 \\
\hline 256. Licata & 18 & 13.56 .21 & 4.1 & $979,916.4$ &, 926.1 & 1.3 & 0.3 & & 0.1 & - 8.4 & $-8 . \overline{1}$ \\
\hline 257. S. Pietro & 15 & 14.29 .45 & 286 & ,928.9 & 926.1 & 88.3 & 26.6 & & 0.0 & +91.1 & $\div 61.4$ \\
\hline 258. Cant. Filozing. & 13 & 39.16 & 377 &, 937.0 &, 926.0 & 116.3 & 39.9 & & 0.5 & 127.3 & 87.9 \\
\hline 259. Cant. M. Grosso & 0.5 & 59.42 & 629 &, 911.5 & .925 .1 & 194.1 & 66.6 & & 0.6 & $130 . \overline{3}$ & 114.6 \\
\hline 260. Floridia & 0.4 .53 & 15.09 .38 & 103.2 & $980,024.5$ &, 924.1 & 31.3 & 10.9 & & 0.6 & 132.3 & 121.9 \\
\hline 261. Gela & & 14.15 .11 & 9.5 & 979 & 9.923 .0 & & 0.7 & $7^{-}$ & 0.1 & -25.5 & +24.9 \\
\hline 262. Siracu & 05 & 15.16.54 & 4.8 & 980.053 .0 &, 922.9 & 1.5 & 0.1 & $T$ & 3. & 131.5 & 134.7 \\
\hline 263. Giarratana & 03.49 & 14.46 .53 & 603.2 & 979.912 .2 &, 922.5 & 186.1 & 66.4 & & 0.7 & 175.3 & 110.1 \\
\hline 261. Buscemi & 48 & 52.50 & 550.8 &, 931.3 &, 922.5 & 170.0 & 60.6 & & 0.6 & 178.7 & 118.7 \\
\hline
\end{tabular}


E. MEU1 - C. MORELL1

asegue TAB. $\mathrm{V}$

\begin{tabular}{|c|c|c|c|c|c|c|c|c|c|c|}
\hline \multirow{2}{*}{ N. Stazione } & \multirow{2}{*}{ " ? } & \multirow{2}{*}{$\begin{array}{c}\lambda . \\
\text { Gipeenw ch) } \\
0 . "\end{array}$} & \multirow{2}{*}{$\begin{array}{c}l_{1} \\
(m)\end{array}$} & \multirow{2}{*}{$\stackrel{\stackrel{g}{ }}{(g a l)}$} & \multirow{2}{*}{$\begin{array}{l}\gamma_{0}^{\prime} \\
(g a l)\end{array}$} & \multicolumn{3}{|c|}{ Correzioni (mgal) } & \multicolumn{2}{|c|}{ Anomalie ( $m$ gal } \\
\hline & & & & & & $0_{5}$ & calotta & $g^{\prime}-g$ & $g_{0} \gamma_{0}^{\prime}$ & $g^{\prime \prime} 0 \cdot \gamma_{0}^{\prime}$ \\
\hline 265. Cauicattini & 37.01 .59 & 15.03 .58 & 362 & $979,963.0$ & $979,919.9$ & +111.7 & -38.3 & $+\quad 0.3$ & +154.8 & +116.8 \\
\hline 266. Acate & & 14.29 .40 & 199 &, 950.1 &, 919.3 & 61.4 & 18.5 & 0.5 & 92.5 & 74.5 \\
\hline 267. Pedalino & 16 & 34.41 & 228 &, 956.7 & ,918.8 & 70.4 & 21.2 & 0.1 & 108.2 & 87.0 \\
\hline 268. Chiaramonte G. & 02 & 42.52 & 846.1 &, 850.0 & ,918.5 & 261.1 & 89.5 & 1.3 & 192.6 & 104.9 \\
\hline 26\%. S. Teresa Long. & 00.19 & 15.14 .50 & 16.8 & $980,0.41 .2$ & ,917.5 & 5.2 & 1.6 & 1.2 & 128.9 & 128.6 \\
\hline 270. Acate Stazione & 36.59 .45 & 14.25 .36 & 100.7 & $979,961.3$ &, 916.6 & 31.1 & 8.9 & 0.0 & 75.7 & 66.8 \\
\hline 2¡1. Castelluccio & 36.59 .01 & 14.56 .33 & 602 & $979,908.7$ & $979,915.6$ & +185.8 & 63.7 & +0.4 & +178.9 & -115.5 \\
\hline 2i2. Balata di Mod. & 58.41 & 49.37 & 498 &, 932.3 & ,915.1 & 153.7 & 52.7 & 0.1 & 170.9 & 118.3 \\
\hline 273. Noto Antica & 57.48 & 15.02 .11 & 437 &, 9.10 .8 & ,913.8 & 134.8 & 46.2 & 0.3 & 161.8 & 115.9 \\
\hline 27. Cassibile & 44 & $11.3+$ & 29.1 & $980,031.5$ &, 913.7 & 9.0 & 2.8 & 0.7 & 126.8 & 124.6 \\
\hline $27.5 . \quad$ it & 28 & 14.31 .35 & 168.8 & $979,965.8$ & ,913.3 & 52.1 & 15.7 & 0.0 & .5 & 88.8 \\
\hline 276. Comiso & 56.42 & 36.09 & 167.8 &, 973.3 &, 912.2 & 51.8 & 17.0 & 1.0 & 112.8 & 96.8 \\
\hline [27\%. Ragusa & 55.06 & 43.36 & 512.7 & ,918.5 &, 909.9 & 158.2 & 54.2 & 0.4 & 166.8 & 113.0 \\
\hline 278. A vola & 54.22 & 15.07 .38 & 54.3 & $980,013.2$ & ,908.8 & 16.7 & 5.1 & 0.6 & 12 & 116.6 \\
\hline 279. Noto & 53.54 & 04.33 & 65.2 &, 008.8 & ,908.2 & 20.1 & 6.3 & 0.4 & 12 & 114.8 \\
\hline 281. Genisi Staz. & 52.56 & 14.37 .29 & 455.9 & $979,915.4$ & ,906.8 & 140.7 & 48.2 & 0.4 & 149.3 & 101.4 \\
\hline 231. Case Migliorisi & 36.52 .22 & 14.29 .23 & 73 & $979,974.3$ & $979,905.9$ & +22.5 & 7.1 & +0.1 & +90.9 & $-1-83.9$ \\
\hline 282. Il Prainito & & 56.25 & 158 &, 989.6 &, 905.6 & 48.8 & 16.7 & 0.1 & 132.8 & 116.2 \\
\hline :3i. Torre De Leva & 02 & 51.41 & 397 &, 938.0 &, 905.5 & 122.5 & 42.0 & 0.1 & 155.0 & 113.1 \\
\hline 284. Modica & 51.05 & 45.17 & 296.5 & $, 949,2$ & ,90.4.1 & 91.5 & 31.4 & 1.6 & 136.5 & 106.8 \\
\hline 285. S. Paolo & 50.21 & 15.01 .31 & 35.2 & $980,014.6$ & ,903.1 & 10.9 & 3.4 & 0.2 & 122.4 & 119.2 \\
\hline 286. S. Croce Cam. & 49.39 & 14.31 .35 & 87 & 979.976 .1 & .902 .1 & 26.8 & 8.1 & 0.1 & 100.9 & 92.9 \\
\hline 237. Roveto Bimm. & 18 & 15.05 .05 & 25.3 & 980.009 .1 & ,901.6 & 7.8 & 1.9 & 0.2 & 115.1 & 113.6 \\
\hline 288. Villa Scucces & 16 & 14.49 .07 & 379 & $979,930.0$ & .901 .5 & 116.9 & 40.1 & 0.2 & 145.4 & 105.5 \\
\hline 289. Rosolini & 48.51 & 57.17 & 109.4 &, 992.6 &, 900.9 & 33.7 & 9.7 & 0.1 & 125.4 & 115.8 \\
\hline 290. Scioli & 47.20 & 42.00 & 94.9 &, 975.0 &, 898.7 & 29.3 & 10.0 & 0.8 & 105.5 & 96.3 \\
\hline 291. Punta Secca & 36.47 .13 & 14.29 .15 & 2 & $979,981.8$ & $979,898.6$ & $+\quad 0.6$ & $-\quad 0.2$ & $-+\quad 0.1$ & +83.8 & +83.8 \\
\hline 2C2. Ispica & 46.38 & 54.57 & 73.1 & .991 .2 &, 897.7 & 22.5 & 7.1 & 0.3 & 116.0 & 109.2 \\
\hline 293. S. Lorenz & 19 & 15.04 .46 & 9.0 & $980,006.4$ &, 897.3 & 2.8 & 0.7 & 0.2 & 111.9 & 111.1 \\
\hline 29-4. Case Bonivini & 00 & 01.11 & 33 &, 000.3 & & 10.2 & 3.1 & 0.2 & 113.7 & 110.8 \\
\hline 295. Donnalucata & 45.54 & 1.4.38.44 & 47 & $979,977.9$ &, 896.7 & 14.5 & 3.6 & 0.2 & 95.8 & 02.4 \\
\hline 296. Cozzo Sarvo & 18 & 57.57 & 38 & ,995.3 & ,895.9 & 11.7 & 3.4 & 0.1 & 111.2 & 108.0 \\
\hline 207. Pozzallo & 43.56 & 50.5 .1 & 17.6 & .989 .2 & & 5.4 & 1.3 & 0.2 & 100.8 & 99.7 \\
\hline 298. Sampieri & 53 & 45.16 & 52.9 & ,975.8 &, 893.7 & 16.3 & 4.3 & 0.1 & 98.4 & 94.3 \\
\hline ¡299. Pachino & 42.35 & 15.05.51 & 42.1 & ,999.4 & .891 .9 & 13.0 & 4.5 & 0.3 & 120.6 & 116.1 \\
\hline
\end{tabular}


Nel quadro delle anomalie positive sono da notare ancora i due massimi locali nella zona di Sciacca ed a $W$ di Castelvetrano: questi corrispondono ad un notevole relativo avvicinamento alla superficie del substrato denso. Va inoltre segnalata la completa eliminazione del massiccio effusivo dell'Etna: ciò starehbe ad indicare che esso costituisce una manifestazione superficiale limitata agli strati più esterni della crosta.

In conclusione, possiamo dire che il rilievo gravimetrico della Sicilia di cui qui si è reso conto, ha consentito di determinare con precisione l'andamento delle isoanomale, e ciò ha portato ad importanti induzioni sulle strutture geologiche profonde e sulla tettonica dell'Isola.

In particolare, sembrerelhe confermata la sintesi tettonica del Beneo, e ciò costituisce, oltre che un notevole apporto dal punto di vista scientifico, anche un sostanziale contributo all'impostazione pratica delle ricerche geominerarie e petrolifere nell'Isola, per le quali una chiara risoluzione d'insieme del problema geologico-tettonico forma una premessa fondamentale ed indispensabile.

Roma - Istituto Nazionale di Geofisica - Maggio 1952.

\section{RIASSUNTO}

Il rilievo gravimetrico della Sicilia ì stato eseguito con due gravimetri II orden previamente tarati per un ruzionale impiego a scopo geodetico. Esso consta di 299 stazioni. di cui 6 di primo ordine costituiscono un pentagono completo con un punto centrale, che è stuta compensato col metodo dei minimi quadrati: precisione "interna" I $0.1 \mathrm{mgal}$ : precisione "esterna" (Potsdam) †. $0.1 \mathrm{mgal}$. Le stazioni di riferimento del $2^{\circ}$ ordine sono 7 , collegate fra di loro e con quelle del $1^{\circ}$ ordine mediante una rete di triangoli, compensata con metodo speditivo: precisione interna !- $0.2 \mathrm{mgal}$ : precisione nel sistema di Potsdam t $0.5 \mathrm{mgal}$. Le stazioni di detaglio hanno pure una precisione interna di t $0.2 \mathrm{mgal}$, confermata dalle numerose ripetizioni.

I risultati sono compendiati in una carta delle isoanomale secondo Bouguer generalizzate, dalla quale si rileva la presenza in tutta la zona centrale dell'Isola di una vasta anomalia negativa, estendentesi in mare verso Sud e circondata per il resto da anomalie positive, no- 
tevoli soprattutto verso SE. La discussione mostra che probabilmente l'anomalia negativa corrisponde ad un'area di depressione tettonica nella quale sarebbero *colate " le argille scagliose ivi accumulandosi col massimo spessore. Tale depressione è circondata da zone dove il substrato denso si avvicina alla superficie o addirittura affiora; il massimo effetto si ha nel SE dell'Isola. Quasi nessuna traccia rimane nelle isoanomale gravimetriche del massiccio effusivo dell'Etna, di cui verrebbe così provato il carattere superficiale, limitato agli strati più esterni della crosta terrestre.

\section{SUMMARY}

The gravimetric relief of Sicily has been executed with two II orden gravity-meters previously calibrated for geodetic purposes. It consists of 299 stations of which 6 of the first order form a complete pentagon with a central point; this has been compensated by the method of least squares: "internal " precision $\pm 0.1 \mathrm{mgal}$; " external" precision (Potsdam) $\pm 0.4 \mathrm{mgal}$. There are seven reference stations of the second order, connected together and with the first order ones by means of a net of triangles, compensated by a rather speedy method: internal precision $\pm 0.2 \mathrm{mgal}$; precision in the Potsdam system $\pm 0.5 \mathrm{mgal}$. The detail stations have an internal precision of $\div 0.2 \mathrm{mgal}$, confirmed by repeated tests.

The results have been represented on a map of Bouguer genew ralized iso-anomals and shou the presence of a wide negative anomaly in the entire central zone of the Island, extending into the sea touard the south, and for the rest, surrounted by positive anomalies, especially marked toutard the southeast. The discussion shows that probably the negative anomaly corresponds to an area of tectonic depression in which the laminated clays have accumulated with greater thichness. This depression is surrounded by a zone where the dense substratum comes close to the surface, or even rises above it, with a maximum effect in the southeastern part of the Island. Almost no trace remains in the gravimetric isoanomalies of the mas. sive flow of Mount Etna, from which would be proved its superficial character, confined to the outermost layers of the earthcrust. 


\section{BIBLIOGRAFIA}

(1) Monelu C.: Studio del gravimetro Worden $n .50$ e sua applicazione per un rilievo geofisico di dettaglio alle foci del Timavo. Annali di Geofisica, IV, 2, 247-271, Roma 1951.

(2) - : Taratura di due gravinetri Worden e collegamenti europei. Ibidem, IV, n. 4, 493-524, Roma 1951.

(3) -: Primo contributo per una rete gravimetrica fondamentale in Italia. Ibidem, V, 1, 97.124, Roma 1952.

(1) - : Rilievo gravimetrico e riduzione isostatica nell'halia nord-orientale. Tecnica Italiana, Nuova Serie, a. VI, n. 3 e 4, Trieste 1951.

(5) - : La rete geofisica e geodetica in Italia nel suo stato attuale e nei suoi rapporti con la struttura geologica superficiale e profonda. Sez. $2^{\prime \prime}$ : Rete gravimetrica. Ibidem, vol. I, n. 3, Trieste 1946.

(6) Beneo E.: Le possibiliti petrolifere della Sicilia nel quadro di unn più realistica interpretazione della tettonica italiana. Boll. Servizio Geologico d'Italia, IXXII, 1950, $1^{\circ}$ fasc, 19-44.

(7) Fabiani R.: Relazione sull'esplorazione geofisica regionale della Sicilia ese. suita nel triennio 1929-1931. Boll. Assoc. Min. Sic., VIII. n. 5-8, Palermo 1932. 